\title{
Dynamic Axis-Tuned Cells in the Monkey Lateral Prefrontal Cortex during a Path-Planning Task
}

\author{
Kazuhiro Sakamoto, ${ }^{1,2}$ Naohiro Saito, ${ }^{2}$ Shun Yoshida, ${ }^{2}$ and Hajime Mushiake ${ }^{2}$ \\ ${ }^{1}$ Department of Neuroscience, Faculty of Medicine, Tohoku Medical and Pharmaceutical University, Sendai 983-8536, Japan, and ${ }^{2}$ Department of \\ Physiology, Tohoku University School of Medicine, Sendai 980-8575, Japan
}

The lateral prefrontal cortex (IPFC) plays a crucial role in the cognitive processes known as executive functions, which are necessary for the planning of goal-directed behavior in complex and constantly changing environments. To adapt to such environments, the lPFC must use its neuronal resources in a flexible manner. To investigate the mechanism by which IPFC neurons code directional information flexibly, the present study explored the tuning properties and time development of IPFC neurons in male Japanese monkeys during a path-planning task, which required them to move a cursor to a final goal in a stepwise manner within a checkerboard-like maze. We identified "axis-tuned" cells that preferred two opposing directions of immediate goals (i.e., vertical and horizontal directions). Among them, a considerable number of these axis-tuned cells dynamically transformed from vector tuning to a single final-goal direction. We also found that the activities of axis-tuned cells, especially pyramidal neurons, were also modulated by the abstract sequence patterns that the animals were to execute. These findings suggest that the axis-tuned cells change what they code (the type of behavioral goal) as well as how they code (their tuning shapes) so that the IPFC can represent a large number of possible actions or sequences with limited neuronal resources. The dynamic axis-tuned cells must reflect the flexible coding of behaviorally relevant information at the single neuron level by the IPFC to adapt to uncertain environments.

Key words: behavioral planning; lateral PFC; monkey

\section{Significance Statement}

The lateral PFC (IPFC) plays a crucial role in the planning of goal-directed behavior in uncertain environments. To adapt to such situations, the IPFC must flexibly encode behaviorally relevant information. Here, we investigated the goal-tuning properties of neuronal firing in the monkey lPFC during a path-planning task. We identified axis-tuned cells that preferred "up-down" or "left-right" immediate goals, and found that many were dynamically transformed from vector tuning to a final-goal direction. The activities of neurons, especially pyramidal neurons, were also modulated by the abstract sequence patterns. Our findings suggest that PFC neurons can alter not only what they code (behavioral goal) but also how they code (tuning shape) when coping with unpredictable environments with limited neuronal resources.

\section{Introduction}

To achieve a specific goal, one must be consciously aware of the goal and organize concrete actions or action sequences to complete it. In primates, the lateral PFC (lPFC) is thought to play a

\footnotetext{
Received Oct. 1, 2018; revised 0ct. 30, 2019; accepted Nov. 1, 2019.

Author contributions: K.S. and H.M. designed research; K.S. and N.S. performed research; K.S. and S.Y. analyzed data; K.S. wrote the first draft of the paper; K.S. and H.M. edited the paper; K.S. wrote the paper.

This work was supported by Japan Society for the Promotion of Science KAKENHI Grant JP16H06276 (Platform of Advanced Animal Model Support), Ministry of Education, Culture, Sports, Science, and Technology KAKENHI Grant 15 H05879 (Non-linear Neuro-oscillology), and Japan Agency for Medical Research and Development (AMED) Grant JP18dm0207051. We thank Midori Takahashi and Yasuko Sato for technical help.

The authors declare no competing financial interests.

Correspondence should be addressed to Kazuhiro Sakamoto at sakamoto@tohoku-mpu.ac.jp or Hajime Mushiake athmushiak@med.tohoku.ac.jp.

https://doi.org/10.1523/JNEUROSCI.2526-18.2019

Copyright $\odot 2020$ the authors
}

fundamental role in the planning and execution of goal-directed behaviors or executive functions (Duncan, 2001; Tanji and Hoshi, 2008; Passingham and Wise, 2012). Behavioral planning often occurs within uncertain and changeable environments. Thus, the IPFC must adapt to environmental changes by flexibly managing its neuronal resources. However, how this process occurs remains to be investigated.

Electrophysiological studies using monkeys have been shown neuronal activities associated with various aspects of executive functions, such as working memory (Funahashi et al., 1989; Miller et al., 1996; Romo et al., 1999), behavioral goals (Saito et al., 2005; Genovesio et al., 2006, 2012), categories or rules (Freedman et al., 2001; Wallis et al., 2001; Shima et al., 2007; Diester and Nieder, 2008), and action sequences (Averbeck et al., 2002, 2006; Mushiake et al., 2006; Averbeck and Lee, 2007; Shima et al., 
2007). Among them, recent studies have demonstrated that IPFC neurons exhibit the dynamical transformation of stimulus-driven activities into activities that are associated with internal representations of behavioral decisions (Machens et al., 2005; Mante et al., 2013) or actions (Sakamoto et al., 2008, 2013; Katori et al., 2011). It has also been reported that the high-dimensional state space of the population activities of IPFC neurons contain a low-dimensional subspace in which taskrelevant variables are represented (Mante et al., 2013; Rigotti et al., 2013; Murray et al., 2017), which indicated flexible information coding in the IPFC. However, to elucidate how the IPFC flexibly manages its resources in detail, it will be necessary to determine what variables the neurons code as well as how the variables are coded. For example, if the variable encoded by a neuron is associated with a specific direction, examination of the directional tuning properties could be valuable for elucidating the mechanism underlying the activity of the IPFC.

Thus, the present study investigated how monkey IPFC neurons encode multiple directional information during a path-planning task (Mushiake et al., 2001, 2006; Saito et al., 2005; Sakamoto et al., 2008, 2013). The task involved the planning of appropriate cursor movements in a stepwise manner to reach a final goal. A group of neurons in which activity changed according to the goal or direction of the first cursor-movement (immediate-goal neurons) was identified, in addition to another group that solely coded final goals (final-goal neurons). Thereafter, the goaltuning properties of these neurons were examined. Surprisingly, in addition to conventional vector tuned cells, which exhibited a maximum firing rate to a single goal direction, a substantial number of immediate-goal neurons were found to be axis-tuned cells. That is, they preferred "up-down" or "left-right" immediate goals. Furthermore, many axis-tuned cells had dynamic tuning properties, such that axis tuning toward immediate goals was transformed from the vector tuning of final goals. The activities of the axis-tuned cells, especially pyramidal neurons, reflected the immediate goal but also the abstract sequence patterns (Shima et al., 2007). The dynamic properties of axis-tuned cells in this report, which is the first regarding the IPFC in macaques, suggest that the IPFC uses its limited neuronal resources in a flexible manner by changing not only what information they code but also how they code it to adapt to uncertain environments.

\section{Materials and Methods}

All experiments were performed using 2 adult male Japanese monkeys (Macaca fuscata) weighing 6.8 and $7.5 \mathrm{~kg}$, respectively. All experimental protocols were approved by the Animal Care and Use Committee of Tohoku University (Permit \#20MeA-2), and all animal protocols conformed with the National Institutes of Health guidelines for the care and use of laboratory animals, as well as with the recommendations of the Weatherall Report. The 2 monkeys used in the present study had previously participated in published studies, including experiments that in-
D Decomposition of goal tuning

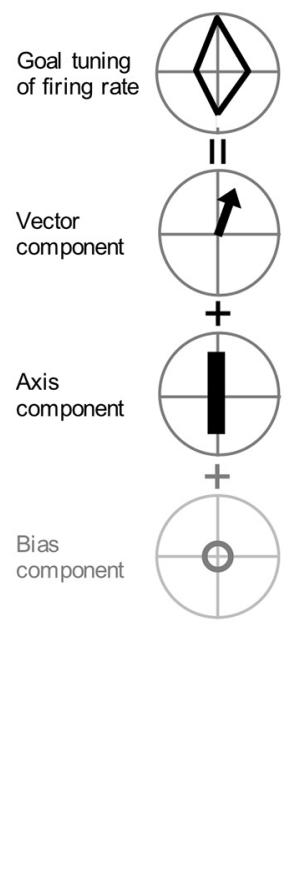

Figure 1. The behavioral task and classification of neurons. $\boldsymbol{A}$, Path-planning task. Successive panels represent each event. The delay period is divided into the first half (Delay 1) and the second half (Delay 2); in the latter-half, the path may be blocked. $\boldsymbol{B}_{\text {, }}$ istribution of spike width for all neurons; interneurons and pyramidal neurons were divided at the valley of the distribution. $\boldsymbol{C}$ Mean firing rates of the putative interneurons and pyramidal neurons. Shaded areas represent SEM. D, Decomposition of goaldirectional tuning for classification of the tuning shape for immediate goals.

vestigated the basic characterizations (Saito et al., 2005; Mushiake et al., 2006) and dynamical properties (Sakamoto et al., 2008, 2013; Katori et al., 2011) of immediate-goal neurons.

Experimental design and behavioral task. During the experiment, each monkey was seated in a primate chair with its head restrained and was oriented toward a computer monitor on which a checkerboard-like maze and a cursor were displayed. Each monkey could operate two manipulanda in the chair by the supination and pronation of either forearm with one degree of freedom to move a cursor through the maze. The supination and pronation of each forearm were assigned to four cursor directions, and eye position was monitored using an infrared eye camera (R21-C-AC; RMS) with a $250 \mathrm{~Hz}$ sampling rate.

Both monkeys were trained to perform a path-planning task (maze task) that has been previously described (Mushiake et al., 2001, 2006; Saito et al., 2005; Sakamoto et al., 2008, 2013) (Fig. 1A). Briefly, they were required to plan multiple movements of the cursor to reach a final goal within the maze. To begin a trial, each animal was required to hold the two manipulanda in a neutral position for $1 \mathrm{~s}$ (Initial hold). After this $1 \mathrm{~s}$ period, a green cursor appeared at the center of the maze to indicate the starting position (Start display); $1 \mathrm{~s}$ later, a red square indicating the position of the final goal within the maze (Final goal display) was displayed for $1 \mathrm{~s}$. After a delay period of $1 \mathrm{~s}$ (Delay 1), one or two of four possible paths to the final goal were blocked in some trials. This was followed by another $1 \mathrm{~s}$ delay (Delay 2), during which the cursor had to be kept at the starting position. Thereafter, the color of the cursor was changed from green to yellow, which served as an initiation signal (the first go). Subsequently, the monkey was required to move the cursor to the first position, which was defined as the immediate goal, within $1 \mathrm{~s}$ and to maintain it there for $1 \mathrm{~s}$ until the cursor became yellow again, which represented the next go signal. The monkey was allowed to move the cursor in any direction toward the next intersection of the maze, except when the path was blocked. When the cursor reached the final goal, the animal was rewarded. 
During the experiment, no visual cues indicating the direction in which the cursor was to be moved were provided while the monkey was moving the cursor in a stepwise manner. Thus, each monkey selected, without the aid of visual cues, the direction for each cursor movement based solely on the memorized position of the final goal. To dissociate hand and cursor movements, the hand-cursor assignments were arranged in three different combinations that changed on the completion of a block of 48 trials (Saito et al., 2005; Mushiake et al., 2006). Each monkey was required to adapt to the new assignments without further instruction; both monkeys required relatively few trials to become accustomed to the new hand-cursor assignments. In $>89 \%$ of trials, the monkeys reached the final goal within the minimum number of three steps.

Neuronal recording. Conventional surgical and electrophysiological techniques were used to obtain in vivo single-cell recordings (Saito et al., 2005; Mushiake et al., 2006; Sakamoto et al., 2008) from the lPFC region, which is located above and below the principal sulcus of the right hemisphere. After the monkeys completed the behavioral training, an acrylic recording chamber was attached to the skull of each monkey under aseptic conditions, and the cortical sulci were identified using an MRI scanner (OPART 3D-System, Toshiba). Before recording neuronal activity in the IPFC, we defined the frontal eye field using intracortical microstimulation; neuronal activities rostral to the frontal eye field, including the banks of the principal sulcus and the adjacent cortical convexity, were recorded.

Neuronal activity was recorded extracellularly using up to four glassinsulated linearly aligned Elgiloy microelectrodes $(1-2.5 \mathrm{M} \Omega$ at $333 \mathrm{~Hz})$ that were manipulated with an electrode positioning system (AlphaOmega, EPS). Individual spikes were amplified with an Alpha-Omega MCP plus 8 and isolated using a template-based discriminator (AlphaOmega, MSD); only well-isolated spikes that were stable over entire recordings and exhibited clear single peaks in the distribution of distance from the template were included in the analysis. The present study focused on neuronal activities during the preparatory period, which included the Start display, Final goal display, and Delay 1 and Delay 2 periods. We previously observed no consistent relationships between eye movement/position and neuronal activity during the analyzed periods (Saito et al., 2005); however, we performed additional analyses on the eye movements to confirm that our new results were not correlated with eye-movement-related parameters. Each neuron was recorded only under the condition of either portrait or landscape configuration (Fig. 2).

Data analysis. The present study examined whether the neuronal activities observed during a 4 s preparatory period (i.e., Start display, Final goal display, Delay 1, and Delay 2) were associated with selectivity for either final goals or immediate goals. For this purpose, a multiple linear regression analysis (Draper and Smith, 1998) assessing spike counts in each $100 \mathrm{~ms}$ time window was conducted using the following formula:

$$
\text { firing rate }=\alpha+\beta \times(\text { final or immediate goals }) \text {, }
$$

where $\alpha$ is the intercept and $\beta$ is the set of coefficients. The categorical factors for the final goals were the four final-goal positions presented in the Final goal display period, whereas those for the immediate goals were the four positions of the cursor at the first step. Therefore, three different dummy variables were used for each of the final goals and immediate goals. Accordingly, $\beta$ included three coefficients. The analyses for the final goals and immediate goals were conducted separately. The $F$ value at each time point was normalized by the significance level of the $F$ value $(p=0.05)$ and was therefore referred to as normalized goal selectivity. Other selectivities, such as "hand used" selectivity, were defined in the same manner. When comparing the relevance of neuronal activities among parameters with different degrees of freedom, such as immediate goal and cursor-movement sequence, adjusted coefficients of determination (ACDs) were adopted.

For the present study, neurons that exhibited immediate-goal selectivity higher than both the significance level and the final-goal selectivity at a certain time period were regarded as having significant immediate-goal selectivity and defined as immediate-goal neurons. These neurons were segregated into two groups based on type as follows: (1) final-toimmediate neurons, which exhibited significant selectivity for the final goal that was higher than the selectivity for the immediate goal at a certain time period (also defined as the significant final-goal selectivity) before significant immediate-goal selectivity (the change from significant final-goal selectivity to significant immediate-goal selectivity was also referred to as a representational shift); and (2) immediate-goal proper neurons, which did not exhibit significant final-goal selectivity. Additionally, final goal neurons that exhibited significant selectivity for the final goals that was higher than the selectivity for the immediate goal throughout the preparatory period were identified. No immediate-tofinal neurons were observed.

Regression analysis was performed on the following behavioral parameters: the hands monkeys used to move the cursor when the first go signal was delivered (hand used); and the cursor-movement sequence, such as up $\rightarrow$ up $\rightarrow$ left (cursor-movement sequence). The number of regressors for the hand used parameter was 1 (i.e., $2-1$ ). The number of regressors for the cursor-movement sequence was $8: 3$ for the immediate goals or the direction of the first cursor movement as described above (Fig. 2, bold blue letters), 2 for the sequence patterns (Fig. 2, purple letters), and 3 for the residual information to reach the final goal, for example, the information of "left" in the case that the final and immediate goals are "left-up" and "up," respectively (Fig. 2, italicized brown letters). The sequence patterns are referred to as the abstract patterns of the cursor-movement sequences. For instance, the patterns of "up $\rightarrow$ up $\rightarrow$ left" and "down $\rightarrow$ down $\rightarrow$ right" are considered the same pattern, "XXY." Using these eight regressors, the relevance of neuronal activities to the cursor-movement sequence could be compared with the immediate goal systematically. In addition, to determine the relationships of firing activities with eye movements and reaction times, correlation analyses were performed for each neuron.

To obtain insights into the underlying mechanisms of neuronal coding, putative interneurons and pyramidal neurons were classified based on the waveforms of their action potentials (Wilson et al., 1994; Rao et al., 1999; Constantinidis and Goldman-Rakic, 2002). To attain a robust degree of distinction, two time distances were obtained from each waveform: (1) the time between the trough and the peak and (2) the time between the beginnings of the initial and the next negative values of the waveform. Dots were plotted on the two-dimensional space of the two time distances, and the norm of one dot from the origin was defined as the spike-width of the neuron. The two time distances had a strong linear correlation $\left(r=0.72, p=4.9 \times 10^{-144}\right)$; thus, the norms were represented by the converted values corresponding to the trough-peak time to confirm their consistency with other related works. Subsequently, an obvious distribution with two peaks was observed (Fig. 1B). Putative pyramidal neurons were defined as neurons with a spike width of $>0.32 \mathrm{~ms}$, whereas interneurons were defined as neurons with a spike width of $<0.32 \mathrm{~ms}$; this criterion is quite consistent with those used in previous studies. The relevance of this distinction was also supported by the observation that the mean firing rate of the interneurons was much higher than that of the pyramidal neurons throughout the preparatory period (Fig. 1C).

The preferred goal was determined for each neuron in each task period in which it had significant final-goal and/or immediate-goal selectivity. The preferred goal was simply defined as the goal for which the neuron showed the highest firing rate in the time window of the highest goal selectivity. For the present study, the preferred goals were referred to as the discrete preferred goals or the discrete preferred directions. To supplement these data, the preferred direction $\left(\theta_{\text {preferred }}\right)$ and other values were calculated for every time window using a Fourier analysis (Wörgötter and Eysel, 1987, 1991) as follows:

$$
\begin{gathered}
\text { Firing Rate }(\theta)=A_{0} \sum_{j=1}^{2}\left[A_{j} \cos (j \theta)+B_{j} \sin (j \theta)\right] \\
\theta_{\text {preferred }}=\arctan \frac{B_{1}}{A_{1}} \\
A m p_{j}=\sqrt{A_{j}^{2}+B_{j}^{2}}
\end{gathered}
$$

where $\theta$ is the direction of the goal. Although somewhat less accurate, the final goals of the right-up, left-up, left-down, and right-down directions were assigned to $45,135,225$, and 315 degrees, respectively, whereas the 
A

\section{Cursor movement sequences}

\section{Portrait configuration}

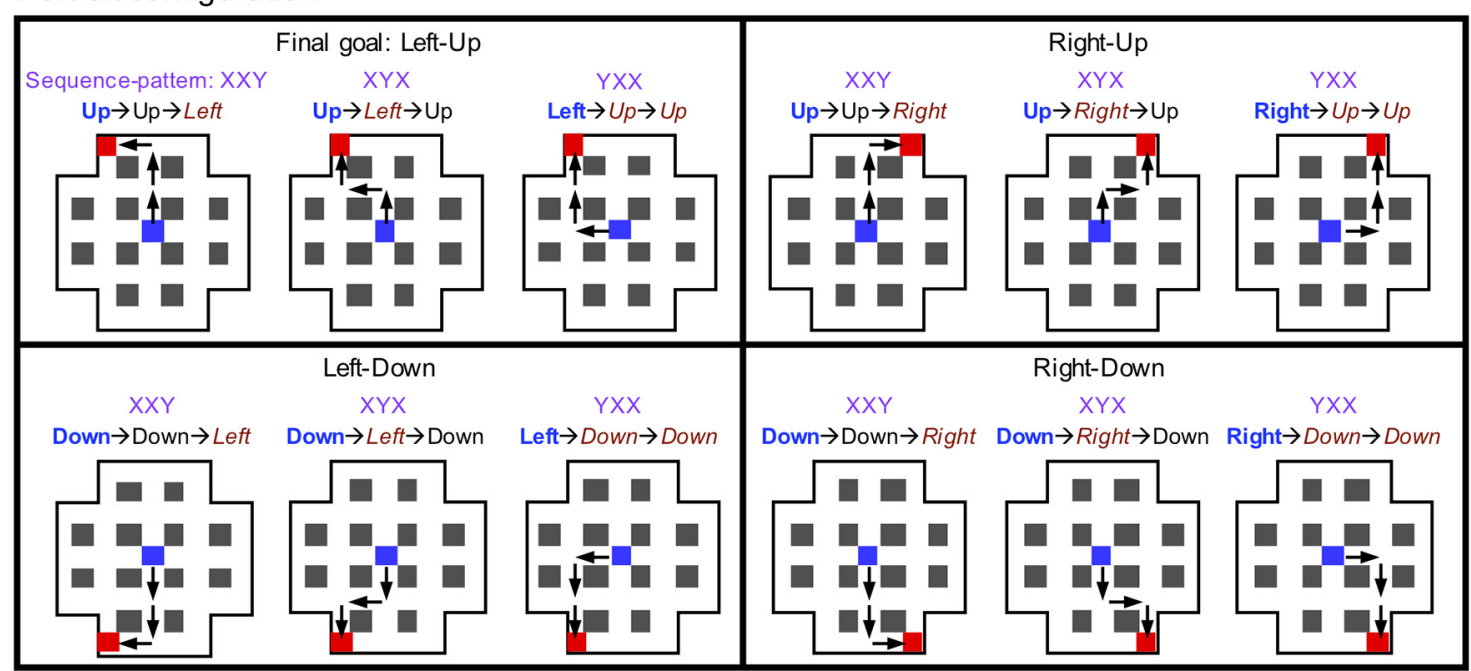

B

Landscape configuration

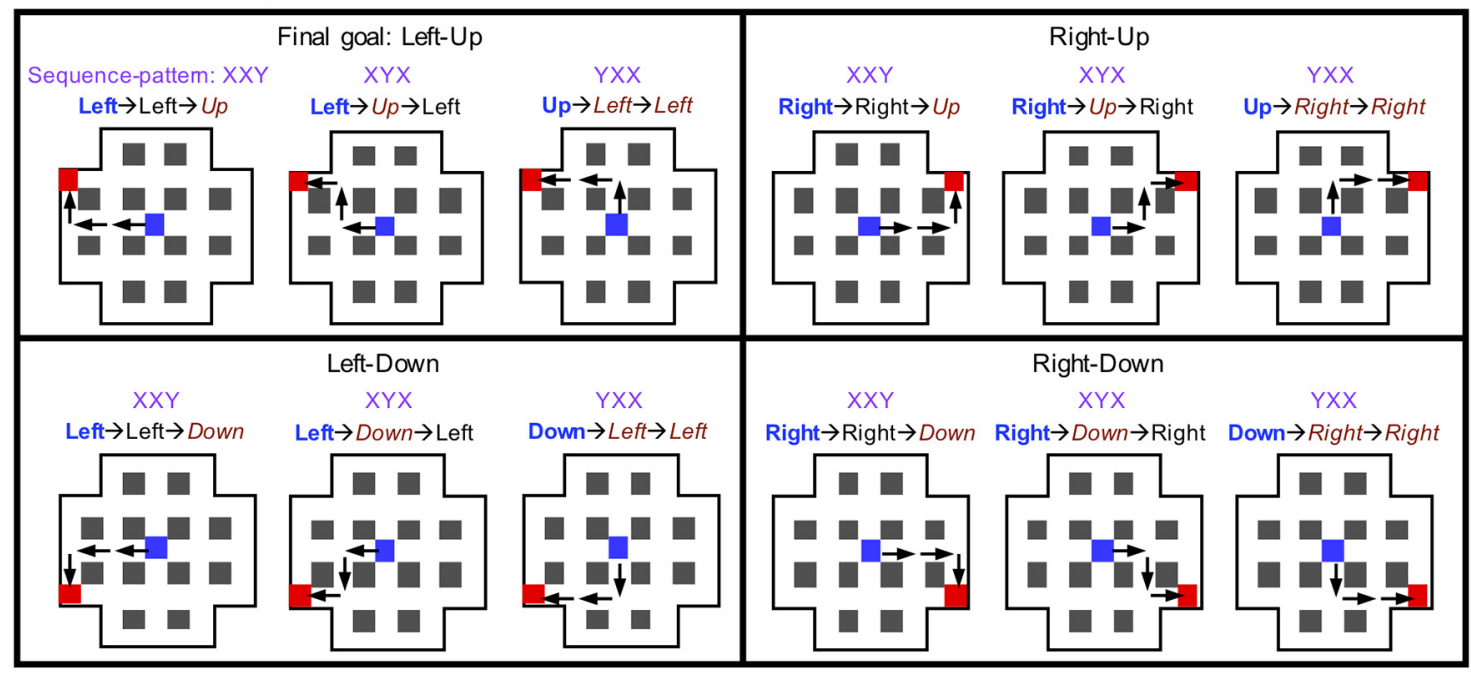

Figure 2. Parameters used in the regression analysis of cursor-movement sequence selectivity. There were two final-goal configurations: the "portrait configuration" $(\boldsymbol{A})$ and the "landscape configuration" $(\boldsymbol{B})$. While one neuron was being recorded, only one final-goal configuration was used. Thus, there are 12 shortest paths for each configuration. These are the only paths that monkeys were able to take when they moved the cursor to final goals using the minimum number of steps. Namely, the trials with back-and-forth cursor movements were not included in the analyses.

immediate goals of the right, up, left, and down directions were assigned to $0,90,180$, and, 270 degrees, respectively. $A_{0}$ was the zero-order component reflecting the bias or average firing rate for the four goals (Fig. $1 D$, "bias component"), and the first harmonic $\left(A_{1}\right.$ and $\left.B_{1}\right)$ was regarded as the directional component; the preferred direction $\left(\theta_{\text {preferred }}\right)$ was obtained from these values (Fig. 1D, "vector component"). For the present study, these directions were referred to as the continuous preferred directions. The second harmonic was considered to be the orientational or axis component (Fig. 1D, "axis component"). However, the angle of the second component, $\arctan \left(B_{2} / A_{2}\right)$, could not be calculated because there were only four goals. Thus, we could only assess whether a given neuron preferred the up-down or left-right direction. The amplitude of the second component was taken as the strength of the axis component. All of these values were calculated separately for the final goals and immediate goals.

Furthermore, to evaluate each neuron's tuning shape, we calculated the axis component to vector component ratio. We classified neurons into two types: if the ratio exceeded 1, the neuron was categorized as axis tuning; otherwise, it was categorized as vector tuning. This ratio was also useful to quantify the degree of axis tuning in each neuron.

Statistical analysis. All statistical analyses were conducted using MATLAB (The MathWorks) or Microsoft Office Excel software. Wilcoxon rank-sum tests or paired $t$ tests were conducted to compare two measures within a group, and $t$ tests were conducted to assess differences in one measure between two different groups. Fisher's exact test was used to verify whether the distribution of neuron classifications differed between interneurons and pyramidal neurons. $p$ values $<0.05$ were considered to indicate statistical significance; unless otherwise specified, all data are presented as a mean \pm SEM.

\section{Results}

Database

In the present study, we recorded the neuronal activities in the IPFC regions of 2 macaque monkeys while they performed a 
Table 1. Numbers of recorded neurons classified according to goal selectivity and cell type

\begin{tabular}{lllllrrrr}
\hline & Total & \multicolumn{2}{c}{ Task-related } & \multicolumn{2}{c}{ Immediate goal } & \multicolumn{2}{c}{ Final goal } \\
\hline Interneurons & 156 & $(18 \%)$ & 85 & $(21 \%)$ & 38 & $(26 \%)$ & 47 & $(18 \%)$ \\
Pyramidal neurons & 731 & $(82 \%)$ & 322 & $(79 \%)$ & 110 & $(74 \%)$ & 212 & $(82 \%)$ \\
Total & 887 & & 407 & & 148 & & 259 & \\
\hline
\end{tabular}

Table 2. Subclassification of immediate-goal neurons

\begin{tabular}{lcccccc}
\hline & & & \multicolumn{2}{l}{$\begin{array}{l}\text { Final-to-imme- } \\
\text { diate }\end{array}$} & \multicolumn{2}{l}{$\begin{array}{l}\text { Immediate- } \\
\text { goal proper }\end{array}$} \\
\hline Interneurons & Total & & 22 & $(27 \%)$ & 16 & $(24 \%)$ \\
Pyramidal neurons & 38 & $(26 \%)$ & 22 & $(73 \%)$ & 51 & $(76 \%)$ \\
Total & 110 & $(74 \%)$ & 59 & & 67 & \\
\hline
\end{tabular}

path-planning task (Fig. 1A; see Materials and Methods); the final goal was reached within the minimum number of steps (i.e., three) on $>89 \%$ of trials (Saito et al., 2005; Mushiake et al., 2006). The analyses only included neuronal activity data recorded during correct trials completed within the minimum number of steps. In total, 887 well-isolated single units were obtained and classified as final-goal or immediate-goal neurons based on the abovementioned criteria using a linear regression analysis. Additionally, these units were classified as interneurons or pyramidal neurons based on spike shape (Table 1; Fig. $1 B$ ). During the preparatory period, 148 immediate-goal neurons exhibited significant selectivity for immediate goals, which were defined as the directions of the cursor movements made by each monkey in the first cursor movement period. Additionally, 259 final-goal neurons that exhibited significant selectivity for final goals but not immediate goals were observed during the same period. The ratio of interneurons to pyramidal neurons, and the difference in the mean firing rates between them, were consistent with accepted values (e.g., Constantinidis and Goldman-Rakic, 2002; Markram et al., 2004) (Fig. 1C). The percentage of interneurons appeared to be slightly higher among immediate- versus final-goal neurons, but this difference was not statistically significant ( $p=$ 0.072, Fisher's exact test).

In this paper, we focus on the detailed properties of immediate-goal neurons $(n=148)$, unless stated otherwise. A significant number of the immediate-goal neurons showed significant final-goal selectivity before elevation of each immediate-goal selectivity; these were referred to as final-to-immediate neurons. The remaining neurons (i.e., those that exhibited significant immediate-goal, but not final-goal, selectivity) were referred to as immediate-goal proper neurons (Table 2). There was no significant difference in the distribution of final-to-immediate neurons or immediate-goal proper neurons between interneurons and pyramidal neurons ( $p=0.65$, Fisher's exact test).

When considering directional selectivity by firing activity, it seems appropriate to consider vector-type tuning, in which a neuron exhibits the highest activity in one particular direction, and the lowest activity in the opposite direction. However, as detailed below, we found that many immediate-goal neurons showed a large amount of activity in response to a particular immediate goal, but also to the opposite immediate goal. To further examine this tuning type, we classified neurons into axis tuning or vector tuning types, according to the ratio of the axis component to the vector component within a goal tuning curve of firing rate, at the point corresponding to each neuron's highest level of immediate-goal selectivity (see Materials and Methods). If the ratio exceeded 1, the neuron was categorized as axis tuning;
Table 3. Classification of the tuning shapes of immediate-goal neurons for immediate goals

\begin{tabular}{llrrlrrl}
\hline Interneurons & Total & Axis & Vector & Pyramidal neurons & Total & Axis & Vector \\
\hline Final-to-immediate & 22 & 11 & 11 & Final-to-immediate & 59 & 24 & 35 \\
Immediate-goal proper & 16 & 9 & 7 & Immediate-goal proper & 51 & 20 & 31 \\
Total & 38 & 20 & 18 & Total & 110 & 44 & 66 \\
\hline
\end{tabular}

otherwise, it was categorized as vector tuning. We counted the numbers of neurons that were axis tuning or vector tuning for immediate goals, for both final-to-immediate neurons and immediate-goal proper neurons (Table 3). Axis tuning characteristics appeared to be more common in the interneurons than in the pyramidal neurons, but this difference was not statistically significant ( $p=0.18$, Fisher's exact test).

\section{Example of interneurons showing a shift from the vector} tuning of final goals to the axis tuning of immediate goals Figure 3 shows a typical example of the activity of interneurons classified as immediate-goal neurons. The firing rate of the neuron in this example was modulated by the direction of the final goal displayed in the Final goal display period, as well as by the immediate-goal directions in the Delay 2 period (i.e., in the latter half of the delay period in which some paths may be blocked). This neuron exhibited the highest firing rate for the left-up final goal in the Final goal display period, and its activity was strongest when the immediate goal was "up" at the end of the Delay 2 period; these properties were quantified using regression analysis (Fig. $3 B$ ). In this neuron, final-goal selectivity increased in the Final goal display period but was followed by a drop in the Delay 1 period and an increase in immediate-goal selectivity during the Delay 2 period. Among the immediate-goal neurons, cells that exhibited shifts in significant and dominant goal selectivity from the final goal to the immediate goal (as shown in Fig. 3) were known as final-toimmediate neurons (Sakamoto et al., 2008). In this study, this type of shift was also referred to as a representational shift.

Figure $3 C$ (left) shows the firing rates associated with four final goals at a point when this neuron exhibited its highest finalgoal selectivity during the Final goal display period (Fig. $3 B$, palered arrow). This goal-directional tuning was regarded as the vector type; more specifically, the left-up goal was the highest, whereas its opposite, the right-down goal, was the lowest. In contrast, the tuning of the firing rates associated with four immediate goals at a point when this neuron exhibited the highest immediate-goal selectivity in the Delay 2 period (Fig. $3 B$, paleblue arrow) had a different tuning shape; the neuron primarily preferred the up goal as an immediate goal. Surprisingly, the opposite direction (i.e., the down goal) was the second preferred immediate goal. This type of tuning shape was distinguished as the axis type of goal-directional tuning (Fig. $3 C$, middle). This characteristic is also seen in Figure $3 C$ (right), which plots the final-goal tuning curves for up-down and left-right immediate goals separately at the peak time of immediate-goal selectivity; this shows that the final-goal tuning of the up-down case of the immediate goal significantly surpasses that of the left-right case for every goal (right-up: $t_{(31)}=5.3, p=8.3 \times 10^{-6}$, Cohen's $d=$ 2.1; left-up: $t_{(33)}=7.0, p=6.0 \times 10^{-8}$, Cohen's $d=2.6$; leftdown: $t_{(34)}=3.1, p=0.042$, Cohen's $d=1.1$; right-down: $t_{(32)}=$ $4.1, p=0.00028$, Cohen's $d=1.7, t$ test $)$.

The time developments of the vector component within the firing rate were calculated for both the final goals and immediate goals (Fig. 3D). When the final-goal selectivity was highest (Fig. $3 D$, pale-red arrows, as in Fig. $3 B$ ), the vector component of the 
A

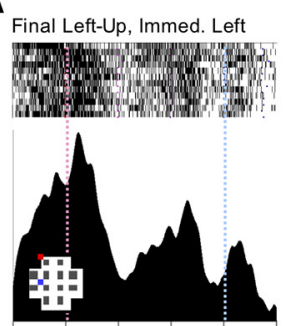

Final Left-Down, Immed Left
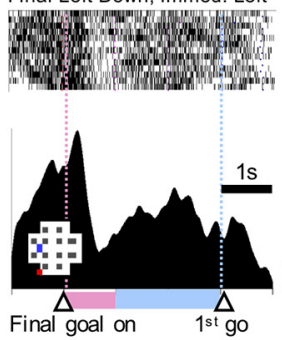

B

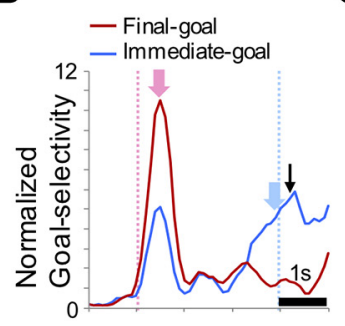

Final-to-immediate neuron (interneuron)
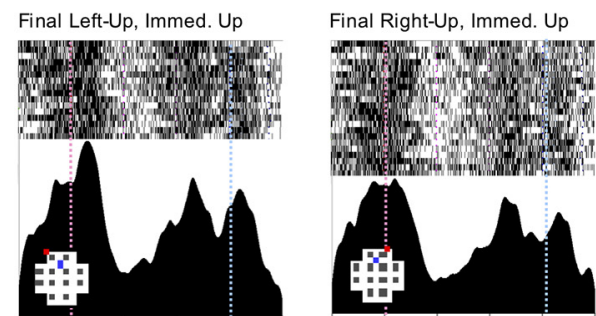

Final Right-Up, Immed. Right
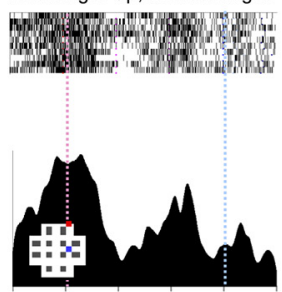

Final Left-Down, Immed. Down

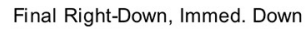

Final Right-Down, Immed. Right
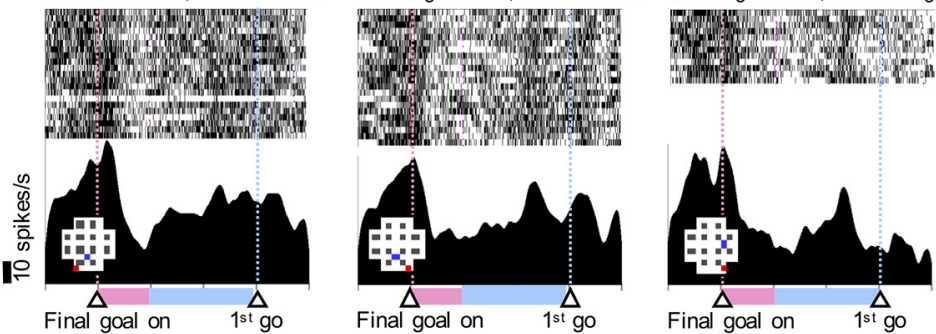

C

Final-goal tuning in Final-goal display

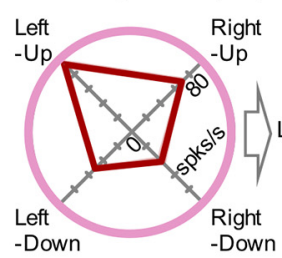

E
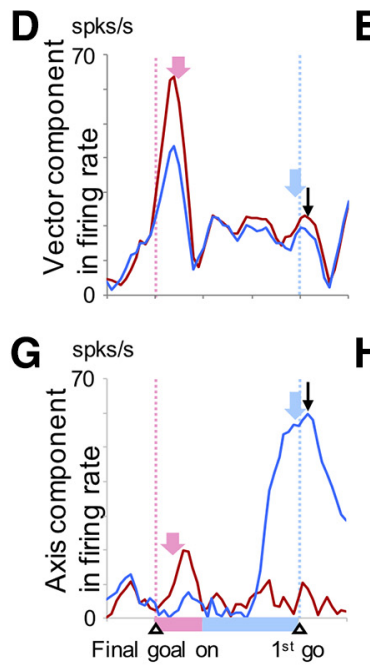

II

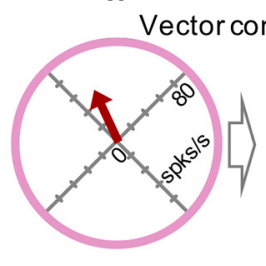

component

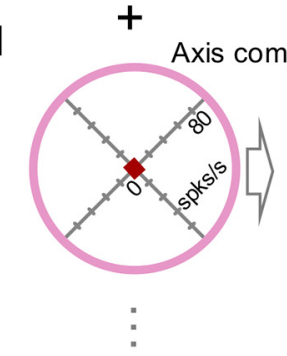

Immediate-goal tuning in Delay 2

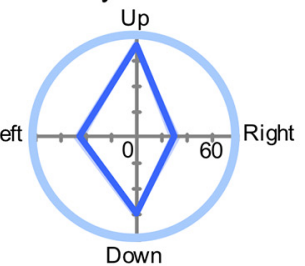

Final-goal tuning in Delay 2

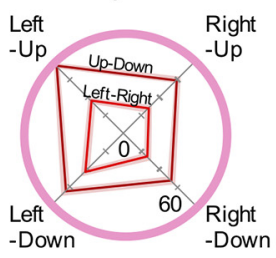

II

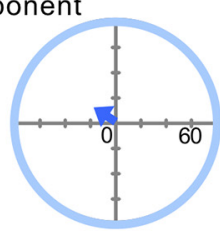

F.
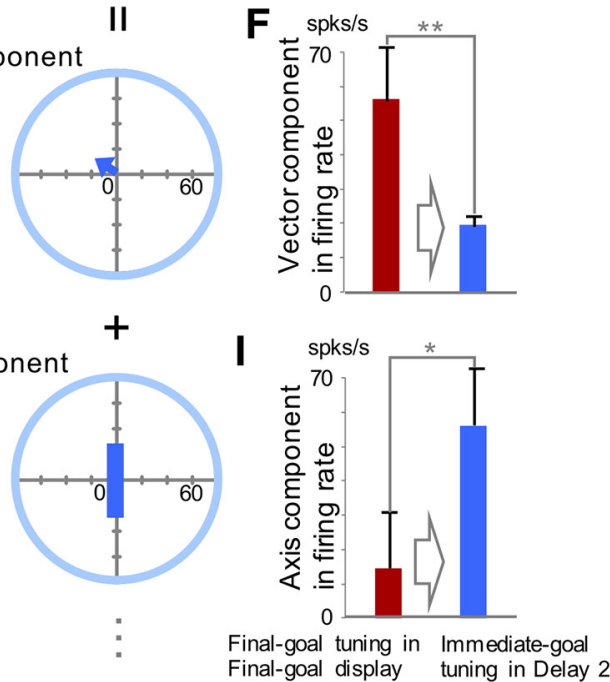

Figure 3. Representative example of an immediate-goal and putative interneuron. $\boldsymbol{A}$, Raster plots and spike density histograms of neuronal activity for each combination of final goals and immediate goals. Red and blue squares represent the locations of the final goal and planned immediate goal, respectively. $\boldsymbol{B}$, Time course of the final-goal (red line) and immediate-goal (blue line) selectivity of the neuron. Goal selectivity is the regression coefficient normalized by the $F$ value at a significance level of $p=0.05$. C, The final-goal directional tuning (left) and immediate-goal directional tuning (middle) at the times indicated by the red and blue arrows, respectively, in $\boldsymbol{B}$. The final-goal directional tunings for the up-down and left-right immediate goals are also shown separately, at the time indicated by the blue arrow (right). Shaded areas represent SEM. $\boldsymbol{D}$, Time-resolved amplitudes of the vector components in firing rate for the final goals and immediate goals. Red and blue arrows are the same as in $\boldsymbol{B}$. $\boldsymbol{E}$, Vector components at the arrows shown in $\boldsymbol{D}$. The direction and length of the arrow within each circle indicate the preferred direction and amplitude of the components, respectively. In the left circle, the preferred direction and its amplitude were $115^{\circ}$ and 56 spikes $/ \mathrm{s}$, respectively, whereas they were $147^{\circ}$ and $18 \mathrm{spikes} / \mathrm{s}$, respectively, in the right plot. $\boldsymbol{F}$, The amplitudes shown in $\boldsymbol{E}$ are replotted. $\boldsymbol{G}, \boldsymbol{H}$, The same plots for the axis components. The amplitudes of the final goal $(\boldsymbol{H}, \boldsymbol{I}$, red bars) and immediate goal (blue bars) are 14 and 58 spikes/s, respectively. ${ }^{*} p<0.05,{ }^{* *} p<0.01$. Black arrows indicate the mean reaction time of the first cursor movement ( $225 \pm 55 \mathrm{~ms}$ ).

final-goal directional tuning was very high, but this component did not show any enhancements in immediate-goal directional tuning when the immediate-goal selectivity was at its highest value (Fig. 3D, pale-blue arrows, as in Fig. 3B). The amplitudes and directions of these vector components at the times of the highest goal selectivity are shown in Figure $3 E$ (left). The amplitude of the final-goal directional tuning in the Final-goal display period was significantly higher than that of the immediate-goal 
directional tuning in the Delay 2 period (Fig. $3 E$, right, $F ; p=$ $7.2 \times 10^{-21}$, Wilcoxon rank-sum test).

Regarding the axis component of the firing rate (Fig. $3 G$ ), the immediate-goal component exhibited a prominent elevation in the Delay 2 period, and the elevation continued until cursor movement onset (black arrow), which was opposite that of the final goals. This elevated component was at its highest value for immediate-goal selectivity (Fig. 3G, pale-blue and black arrows, as in Fig. $3 B, D$ ) and preferred the up-down direction (Fig. $3 \mathrm{H}$, right); its amplitude was significantly higher than that of the axis component for the final-goal selectivity during the highest finalgoal selectivity (Fig. $3 H$, left, I; $p=0.041$, Wilcoxon rank-sum test). It is also noteworthy that the time profile of the final-goal vector component around its peak (Fig. $3 D$, red line) resembles the profile of final-goal selectivity (Fig. 3B, red line), whereas elevation of the immediate-goal axis component in the Delay 2 period (Fig. 3G, blue line) seems to correspond to that of immediate-goal selectivity (Fig. 3B, blue line).

\section{Examples of pyramidal neurons showing axis tuning for immediate goals}

Figure 4 depicts the goal-directional tuning properties of a representative pyramidal neuron classified as a final-to-immediate neuron in the same format as Figure 3; Figure $4 A$ illustrates its neuronal activity. The firing rate was high when the left-down final goal was presented in the Final goal display period, and this elevated activity was sustained after the final goal was turned off. In contrast, during the Delay 2 period, relatively high neuronal discharge rates were observed for the left immediate goal (i.e., in cases in which the monkey moved the cursor to the left after the go signal). It should be noted that, during the same period, this neuron exhibited considerably high activity for the right immediate goal, which was opposite to the neuron's preferred immediate goal, whereas it exhibited its lowest activity for the up immediate goal.

Figure $4 B$ shows a plot of the time development of the goal selectivity in this neuron, which switched its selectivity from final goal to immediate goal after showing persistent final-goal selectivity throughout the Final goal display and Delay 1 periods. To compare these findings with the goal-directional tunings of the interneuron shown in Figure 3, Figure 4C (left, middle) indicates the goal-directional tunings at the peak of final-goal selectivity in the Final goal display period (Fig. $4 B$, pale-red arrow) and at the peak of immediate-goal selectivity in the Delay 2 period (Fig. $4 B$, pale-blue arrow). Consistent with the data shown in Figure $4 A$, final-goal directional tuning during the Final goal display period was prominent for the left-down direction (Fig. 4C, left). In contrast, immediate-goal directional tuning during the Delay 2 period exhibited an axis tuning shape (Fig. $4 \mathrm{C}$, middle), as in the case of the interneuron presented in Figure 3. We also obtained separate, simultaneous final-goal directional tunings (Fig. $4 C$, right) for up-down and left-right immediate goals. The tuning to the left-right immediate goals surrounded that to the up-down immediate goals, confirming the existence of axis tuning (rightup: $t_{(25)}=5.2, p=2.2 \times 10^{-5}$, Cohen's $d=1.8$; left-up: $t_{(25)}=$ $5.5, p=9.6 \times 10^{-6}$, Cohen's $d=2.3$; left-down: $t_{(28)}=1.5, p=$ 0.15 , Cohen's $d=0.53$; right-down: $t_{(26)}=2.5, p=0.018$, Cohen's $d=0.96, t$ test).

The characteristics of the vector components are displayed in Figure $4 D-F$, and comparisons of the axis components are shown in Figure $4 G-I$. Consistent with the interneuron example in Figure 3 , the axis component of the immediate goals increased in the Delay 2 period (Fig. $4 G$ ), and its amplitude was highest for peak immediate-goal selectivity (Fig. $4 B, D, G$, pale-blue arrows); this amplitude was higher than its final-goal counterpart during the Final goal display period (Fig. $4 H, I$, pale-red arrows; $p=$ 0.00043 , Wilcoxon rank-sum test). In contrast, the vector component of the final goals was relatively higher than that of the immediate goals (Fig. 4D) during the Final goal display and Delay 1 periods, and its amplitude for the highest final-goal selectivity in the Final goal display period was only slightly higher than that for the corresponding immediate goals in the Delay 2 period (Fig. $4 E, F ; p=0.028$, Wilcoxon rank-sum test). As in the interneuron example in Figure 3, this pyramidal neuron example exhibited similar time-profiles between the vector component of the final goals and the final-goal selectivity as well as between the axis component of the immediate goals and the immediate-goal selectivity around their peaks.

Another typical immediate-goal pyramidal neuron is illustrated in Figure 5. This neuron was classified as an immediategoal proper neuron; namely, this neuron did not show the representational shift from final goal to immediate goal. That is, it did not exhibit significant modulation in its firing rate for the final goals, whereas it had a strong significant selectivity for immediate goals in the Delay 2 period (Fig. $5 A, B$ ). As can be expected from the firing-rate profile shown in Figure $5 A$, prominent axial tuning was observed in the tuning shape for the immediate goals (Fig. $5 \mathrm{C}$ ) at the peak of immediate-goal selectivity (Fig. 5B, pale-blue arrow): this neuron primarily preferred the down immediate goal but also showed significant firing for the up immediate goal. In contrast, almost no firing was observed for the left and right immediate goals. The preference for the up-down goal is also apparent in Figure 5C (right, right-up: $t_{(34)}=3.0, p=$ 0.0054 , Cohen's $d=1.2$; left-up: $t_{(31)}=4.3, p=0.00017$, Cohen's $d=1.7$; left-down: $t_{(33)}=6.0, p=1.0 \times 10^{-6}$, Cohen's $d=2.6$; right-down: $t_{(33)}=4.1, p=0.00027$, Cohen's $d=1.6, t$ test $)$.

The properties of the vector components (Fig. $5 D-F$ ) and the axis components (Fig. 5G-I) were also examined. A comparison of the data in Figure 5B, $G$ demonstrated that the time-resolved axis component of the immediate goals exhibited good correspondence with that of immediate-goal selectivity. In this example, it was not surprising that both the vector and the axis components of the immediate goals at the peak of immediategoal selectivity were significantly higher than the corresponding components of the final goals (Fig. $5 E, F ; p=7.7 \times 10^{-6}$; Fig. $5 H, I ; p=3.7 \times 10^{-12}$, Wilcoxon rank-sum test). However, a prominent increase in the axis component for the immediate goals was evident, and the axis component remained relatively high, although it showed a partial decrease at a point close to the execution of the first cursor movement (Fig. 5G, black arrow).

\section{Axis components in the immediate-goal tuning increase} in the Delay 2 period in interneurons and pyramidal neurons The average final-goal and immediate-goal selectivity values of the immediate-goal neurons exhibited a representational shift from the final goal to the immediate goal (Fig. 6A, $B$ ), whereas the pyramidal neurons and interneurons exhibited distinctive time profiles for goal selectivity. The final-goal selectivity of the interneurons decreased during the Delay 1 period (Fig. 6A, red line), whereas that of the pyramidal neurons maintained a high value during the same period (Fig. $6 B$, red line). Following a surge in the Final goal display period, these two values significantly differed in the middle of the Delay 1 period (maximum difference, $400-500 \mathrm{~ms}$ from the Delay 1 start: $t_{(146)}=2.1, p=0.036$, Cohen's $d=0.48, t$ test), although both types of neuron exhibited two peaks associated with goal selectivity: one peak was the final 
A

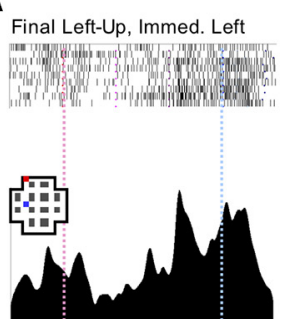

Final Left-Down, Immed. Left mond

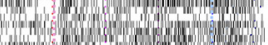

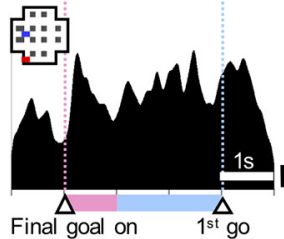

B
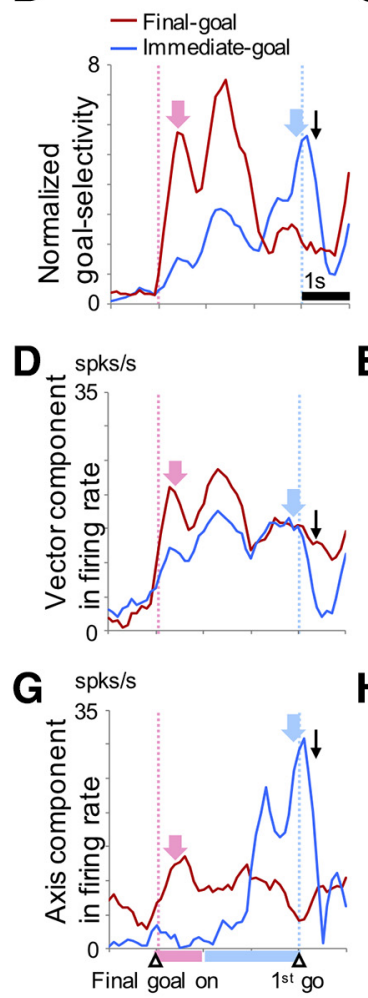

Final-to-immediate neuron (pyramidal neuron)
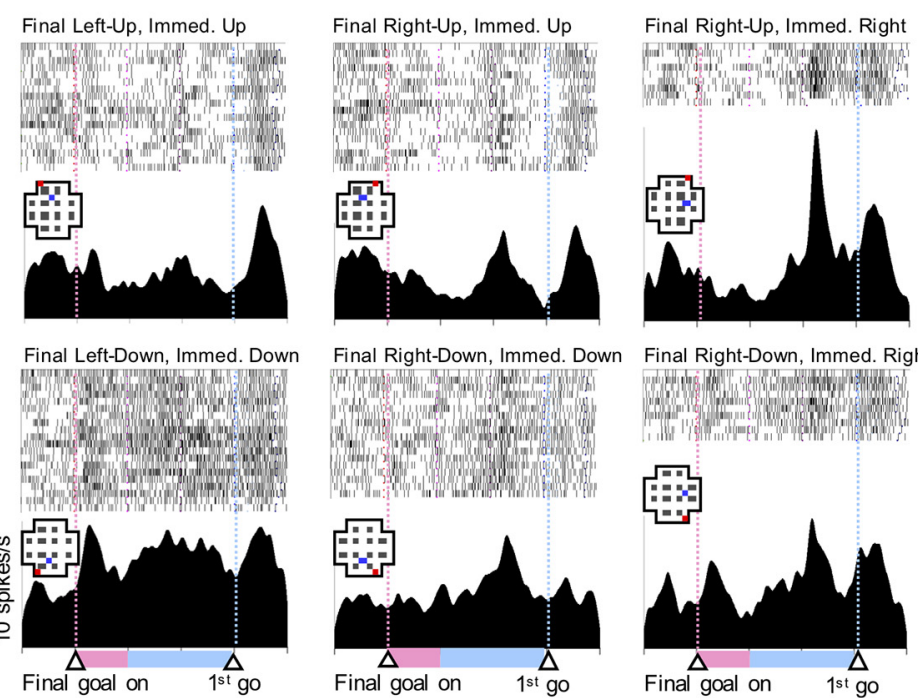

Final Right-Down, Immed. Down

Final Right-Down, Immed. Right
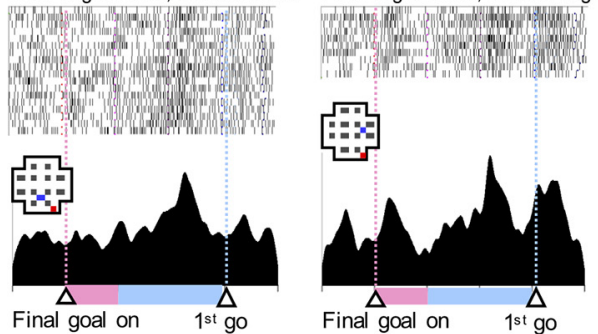

C

Final-goal tuning in Final-goal display

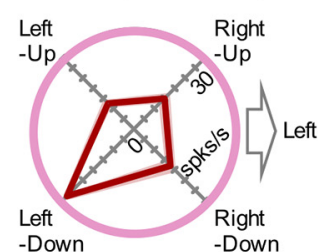

E

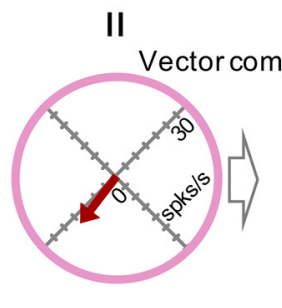

H

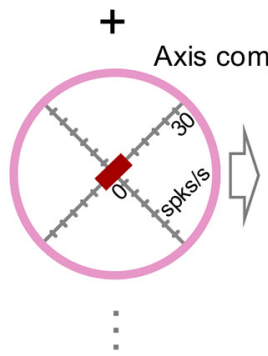

Immediate-goal tuning in Delay 2

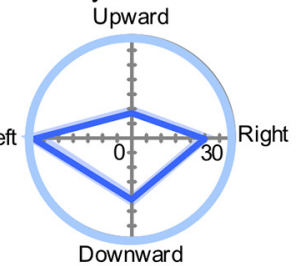

Final-goal tuning in Delay 2

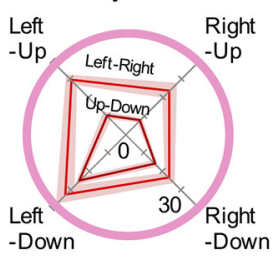

F spks/s

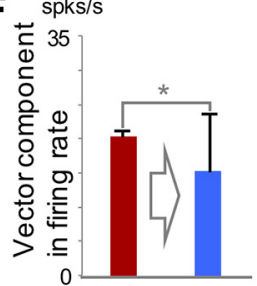

I
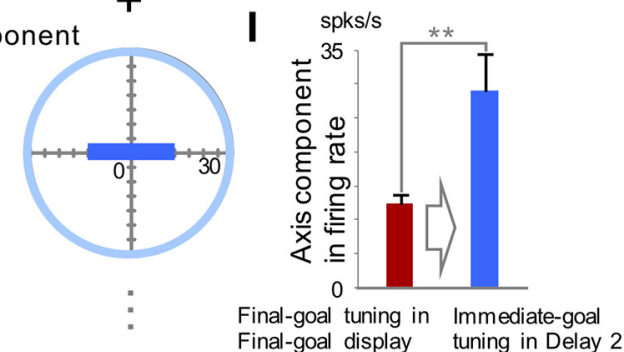

Figure 4. Representative example of an immediate-goal and putative pyramidal neuron. This neuron exhibited a representational shift from the final goals to the immediate goals. The figure formats are the same as those in Figure 3. A, Raster plots and spike density histograms for each combination of final goals and immediate goals. $\boldsymbol{B}$, Time evolution of the final-goal (red line) and immediate-goal (blue line) selectivity of the neuron. $\boldsymbol{C}$, Final-goal directional tuning (left) at the times in Final goal display period ( $\boldsymbol{B}$, red arrow), as well as immediate-goal directional tuning (middle) and final-goal directional tuning (right) at the times in the Delay 2 period ( $\boldsymbol{B}$, blue arrow). In the right circle, the tunings for the up-down and left-right immediate goals are illustrated separately. Shaded areas represent SEM. $\boldsymbol{D}-\boldsymbol{F}$, Plots for the vector components. For the final-goal directional tuning at the red arrow in $\boldsymbol{B}$, the preferred direction and its amplitude were $233^{\circ}$ and 18 spikes $/$, respectively, whereas the preferred direction and amplitude at the blue arrow for the immediate goals were $234^{\circ}$ and 15 spikes $/$, respectively. $G-I$, The same plots for the axis components. The amplitudes for the final goals and immediate goals were 17 and 28 spikes $/$, respectively. ${ }^{*} p<0.05,{ }^{* *} p<0.01$. Black arrows indicate the mean reaction time of the first cursor movement (261 $\pm 77 \mathrm{~ms})$.

goal in the Final goal display period, and the other was the immediate goal in the Delay 2 period. Thus, subsequent analyses of the goal-tuning properties during these two periods were performed.

The time developments of the vector and axis components in the firing rates of the interneurons and pyramidal neurons were also analyzed at the population level for the final goals and immediate goals (Fig. 6C-J). In the interneurons, the vector components of the final goals increased and decreased during the Final goal display and the Delay 1 periods, respectively (Fig. 6C); this was similar to final-goal selectivity. In the pyramidal neu- 
A

\section{Immediate-goal-proper neuron (pyramidal neuron)}

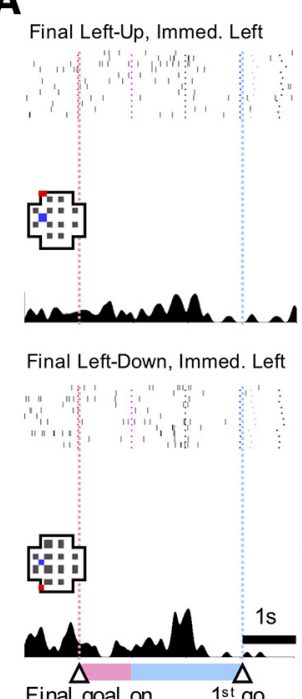

Final goal on $\quad 1^{\text {st }}$ go

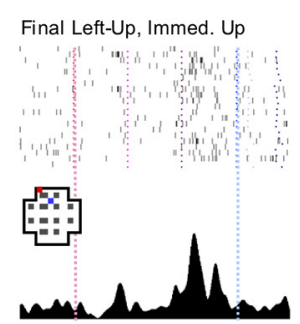

Final Left-Down, Immed. Down
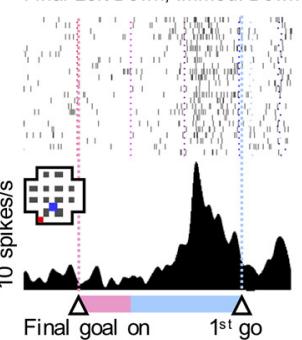

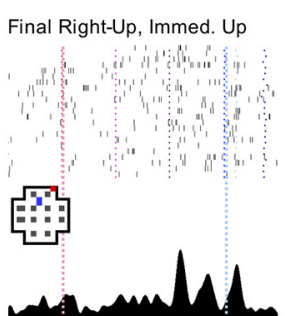

Final Right-Down, Immed. Down
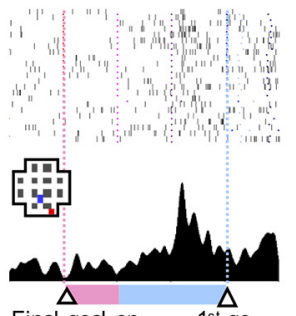

Final goal on $\quad 1^{\text {st }}$ go

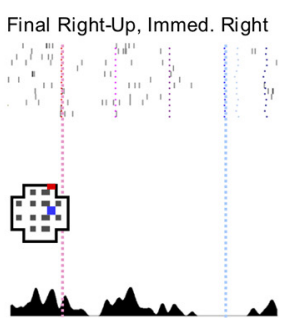

Final Right-Down, Immed. Right

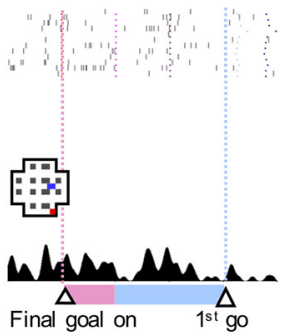

B

C

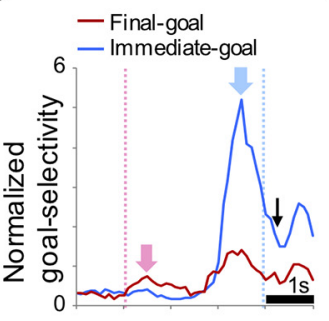

Final-goal tuning in Final-goal display

Immediate-goal tuning in Delay 2
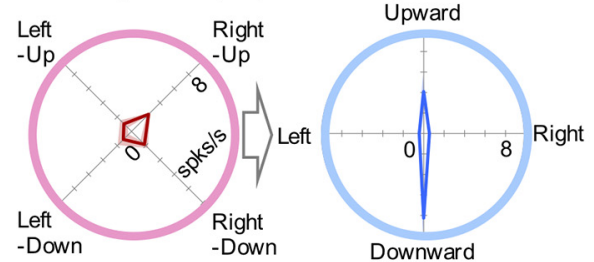

Final-goal tuning in Delay 2
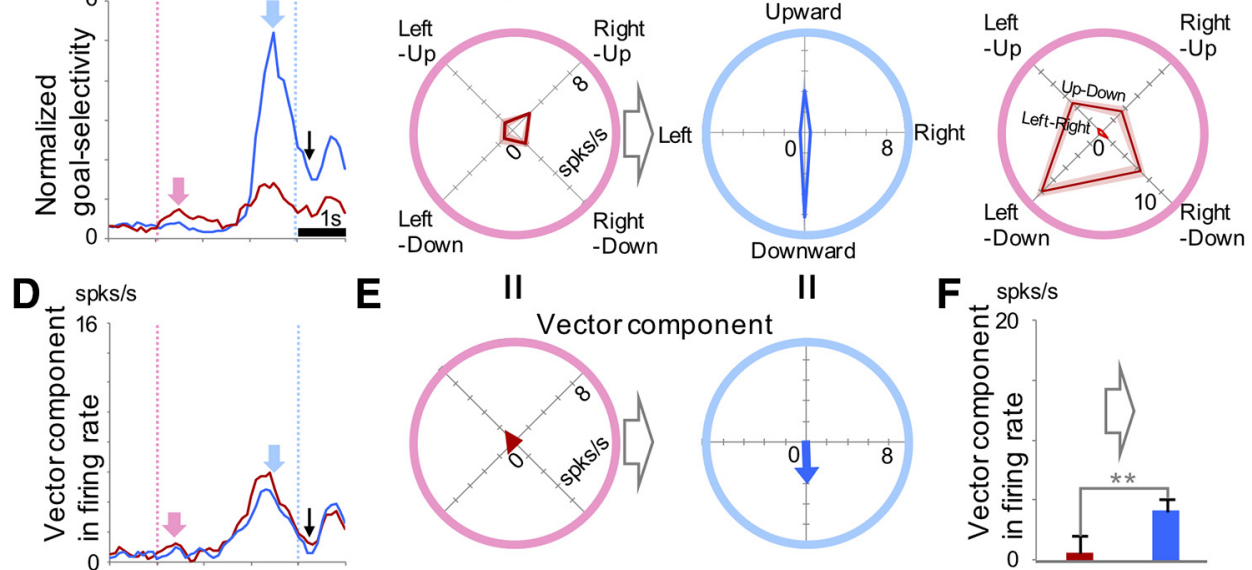

E

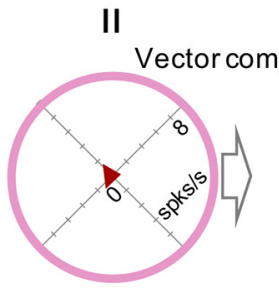

II

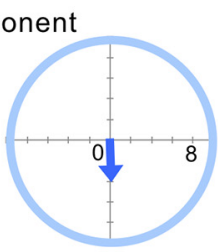

$F_{\text {spks/s }}$
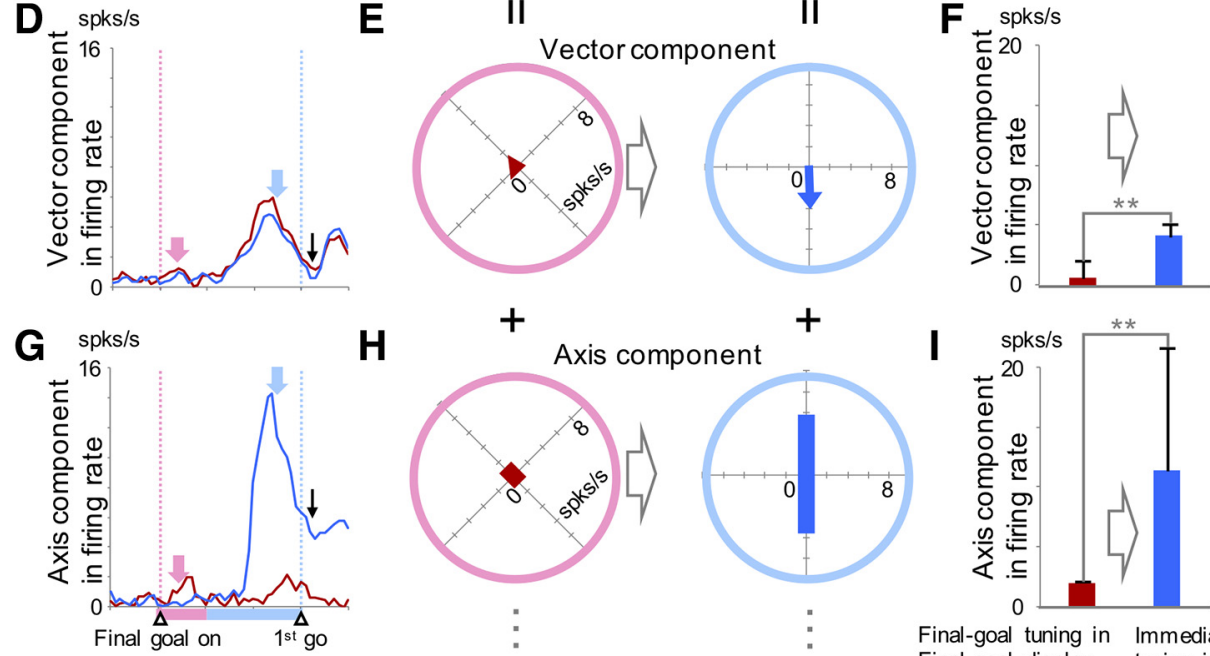

H
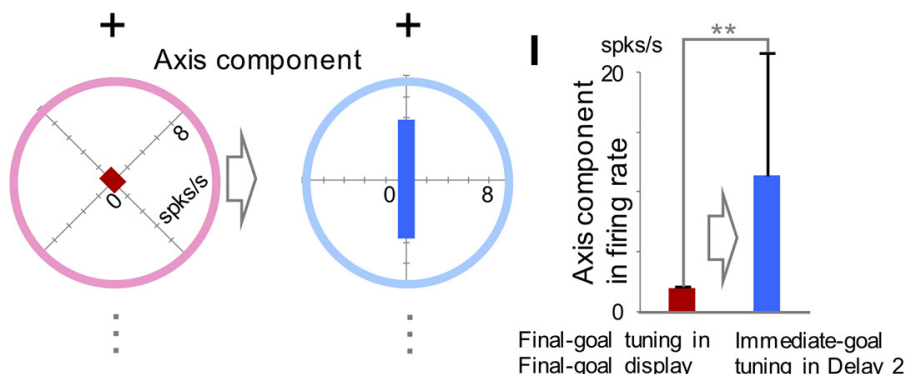

Figure 5. Representative example of an immediate-goal proper and putative pyramidal neuron. $A-I$, The figure formats are the same as those in Figures 3 and 4 . The direction and amplitude of the vector component of the immediate goals were $272^{\circ}$ and 4 spikes $/ \mathrm{s}$, respectively, whereas the amplitude of the axis component of the immediate goals was 11 spikes $/ \mathrm{s}$. ${ }^{* *} p<0.01$. Black arrows indicate the mean reaction time of the first cursor movement ( $237 \pm 58 \mathrm{~ms})$.

rons, the time profile of the vector component was similar to that of final goal selectivity but showed a rather sustained amplitude compared with that of the interneurons (Fig. 6E). The amplitude of the vector component of the final goals at the final-goal selectivity peak in the Final goal display period (pale-red arrows) did not significantly differ from that of the immediate goals in the Delay 2 period (pale-blue arrows) in either interneurons or pyramidal neurons (Fig. $6 D, F$; interneurons: $t_{(36)}=0.41, p=0.68$, Cohen's $d=0.079, r=0.41$; pyramidal neurons: $t_{(108)}=1.0, p=$ 0.30 , Cohen's $d=0.094, r=0.56$, paired $t$ tests). Regarding the axis components, both interneurons and pyramidal neurons exhibited surges in the Delay 2 period (Fig. $6 G, I$ ), and both amplitudes were significantly higher at the peak immediate-goal selectivity in the Delay 2 period than at the peak final-goal selectivity in the Final goal display period (Fig. $6 \mathrm{H}$, J; interneurons: $t_{(36)}=4.2, p=0.00018$, Cohen's $d=0.80, r=0.48$; pyramidal neurons: $t_{(108)}=3.3, p=0.0015$, Cohen's $d=0.32, r=0.47$, paired $t$ tests). The axis component remained sufficiently high around the time of cursor movement onset in both interneurons and pyramidal neurons (Fig. 6G,I, black arrows). Additionally, in 


\section{Immediate-goal neurons}
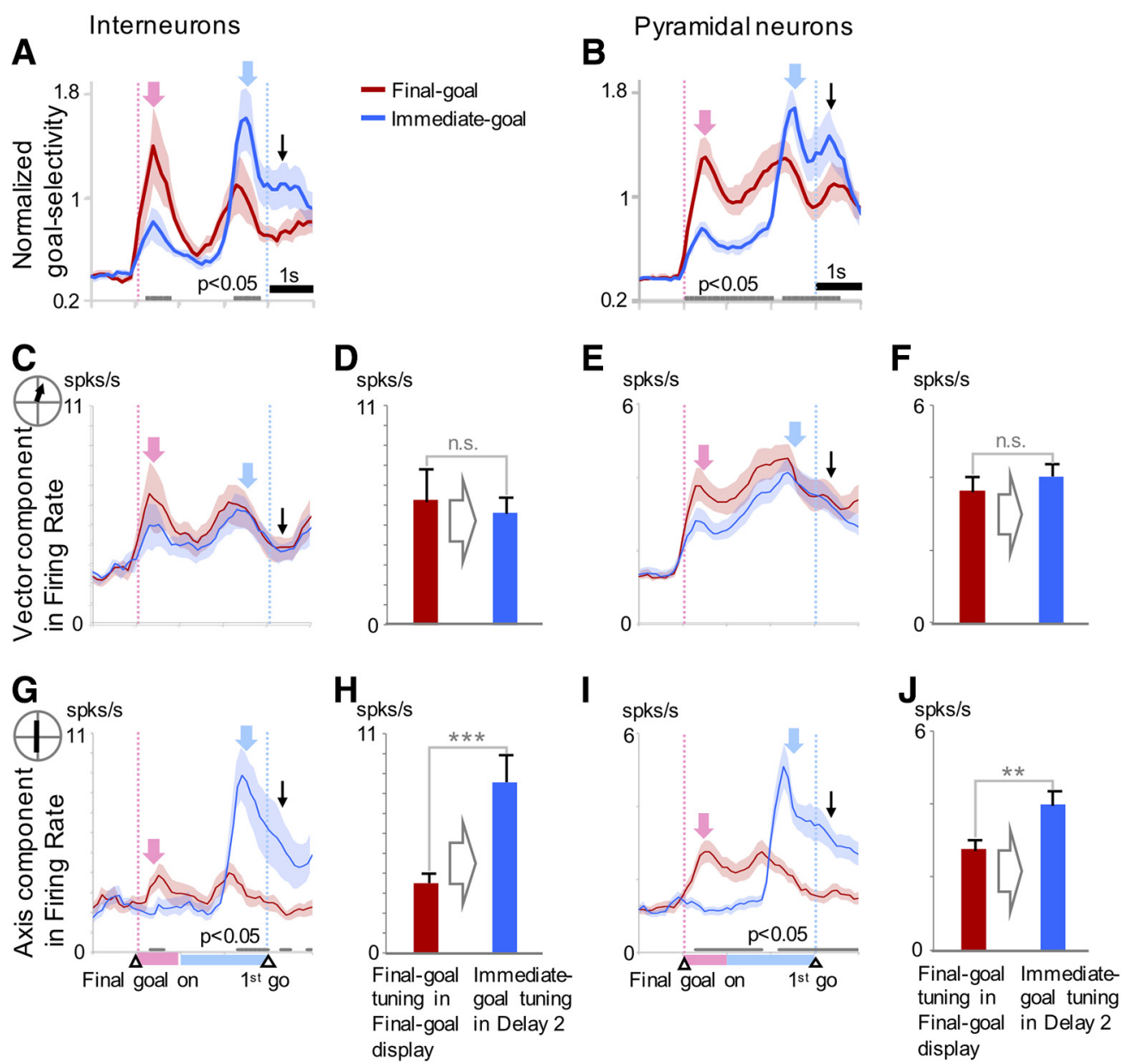

Figure 6. Changes in the final-goal and immediate-goal encoding properties in the immediate-goal neurons at the population level. $\boldsymbol{A}, \boldsymbol{B}$, Time developments of the final-goal and immediategoal selectivity in the populations of $(\boldsymbol{A})$ interneurons and $(\boldsymbol{B})$ pyramidal neurons. Gray bars below the graphs represent time periods in which the final-goal and immediate-goal selectivity significantly differed ( $p<0.05$, Wilcoxon rank-sum test). $\mathbf{C}-\boldsymbol{F}$, Time developments of the amplitudes of the vector components and comparisons of the amplitudes at the peaks of the final-goal and immediate-goal selectivity are indicated by the red and blue arrows in $\boldsymbol{A}$ and $\boldsymbol{B}$, respectively. $\boldsymbol{C}, \boldsymbol{D}$, Interneurons. $\boldsymbol{E}, \boldsymbol{F}$, Pyramidal neurons. The components were calculated for each $100 \mathrm{~ms}$ bin. $\boldsymbol{G}-\boldsymbol{J}$, The same plots for the amplitudes of the axis components. Gray bars represent significant differences: $\mathbf{C}, \boldsymbol{E}, \mathbf{G}, \boldsymbol{I}, p<0.05 ; \mathbf{D}, \boldsymbol{F}, \boldsymbol{H}, \boldsymbol{J},{ }^{* *} p<0.01 ;{ }^{* * *} p<0.001$, n.s. (not significant), $p>0.05$. Black arrows indicate the mean reaction time of the first cursor movement ( $302 \pm 83 \mathrm{~ms}$ ). Shaded areas represent SEM.

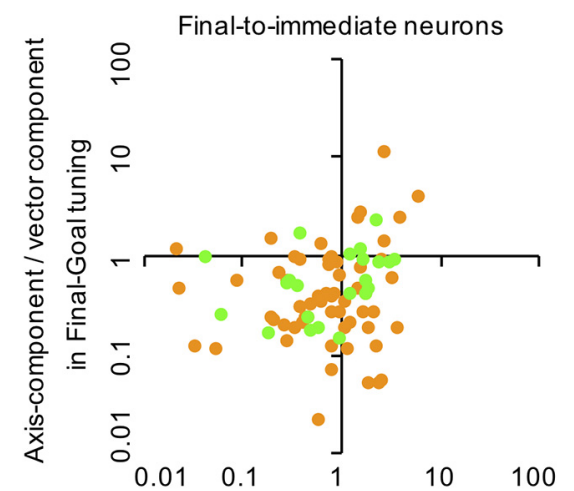

Axis-component/ vector component

in Immediate-Goal tuning

Interneurons Pyramidal neurons

Figure 7. Changes of tuning shape in final-to-immediate neuronal activity. Tuning shape is quantified by the ratio of the axis component to the vector component, in both final-goal tuning (vertical axis) and immediate-goal tuning (horizontal axis), at each neuron's peak selectivity. Interneurons $(n=22)$ and pyramidal neurons $(n=59)$ are distinguished by color.
Table 4. Classification of the tuning shapes of the final goal selectivity of the finalto-immediate neurons in the final goal display period

\begin{tabular}{llcc}
\hline Final-to-immediate & Total & Axis & Vector \\
\hline Interneurons & 22 & 4 & 8 \\
Pyramidal neurons & 59 & 9 & 50 \\
Total & 81 & 13 & 68 \\
\hline
\end{tabular}

both interneurons and pyramidal neurons, the vector components of the immediate goals did not surpass those of the final goals, even at the peak immediate-goal selectivity, whereas the axis component of the immediate goals in the Delay 2 period exhibited a marked increase relative to that of the final goals.

To fully represent the variability and complexity of the data, in addition to the mean data, Figure 7 depicts a scatter plot showing the changes in tuning properties of each neuron. For final-toimmediate neurons $(n=81)$, the tuning shape for final goals was also quantified at each neuron's peak final-goal selectivity. The horizontal and vertical axes of Figure 7 represent the ratio of the axis component to the vector component in the immediate- and final-goal directional tuning, respectively, at the peak time. Al- 
A

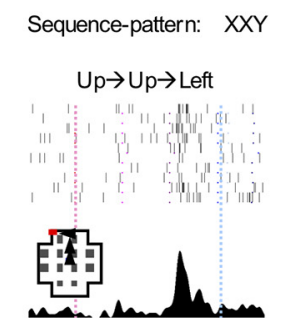

$\mathrm{Up} \rightarrow \mathrm{Up} \rightarrow$ Right
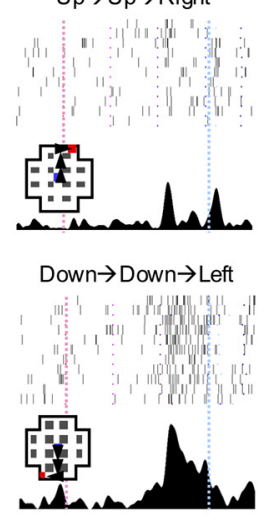

Down $\rightarrow$ Down $\rightarrow$ Right

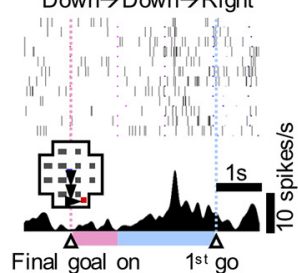

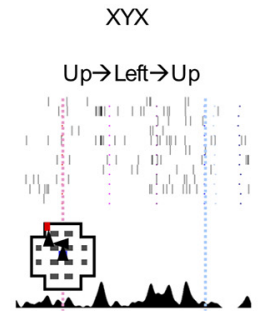

$\mathrm{Up} \rightarrow \mathrm{Right} \rightarrow \mathrm{Up}$

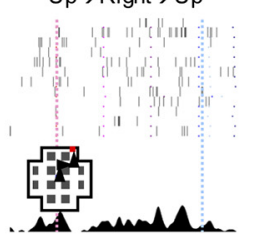

Down $\rightarrow$ Left $\rightarrow$ Down

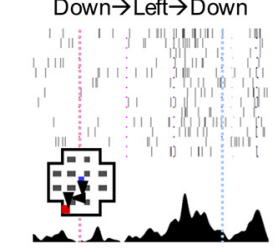

Down $\rightarrow$ Right $\rightarrow$ Down
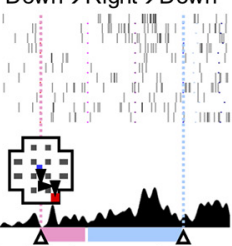

Final goal on

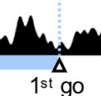

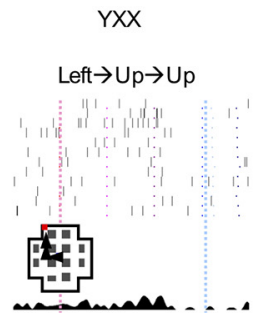

Right $\rightarrow U p \rightarrow \cup p$

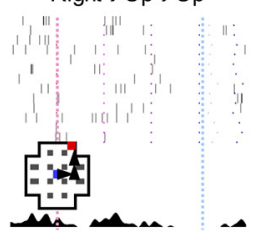

Left $\rightarrow$ Down $\rightarrow$ Down

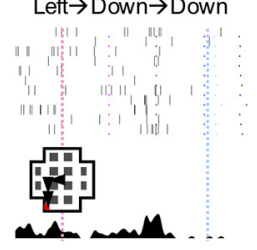

Right $\rightarrow$ Down $\rightarrow$ Down

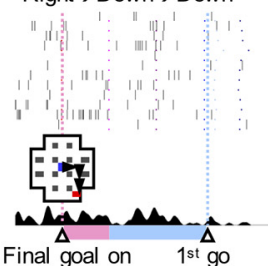

B

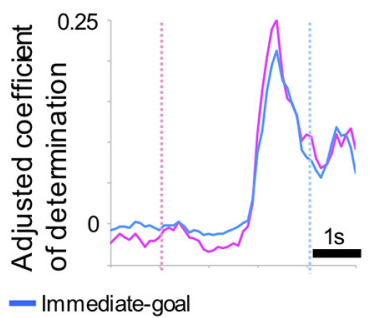

- Cursor-movement-sequence

C

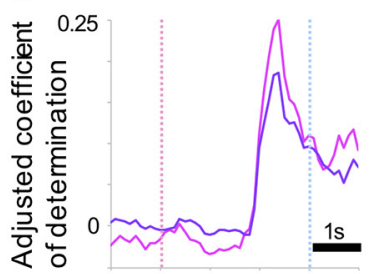

- Sequence-pattern

- Cursor-movement-sequence

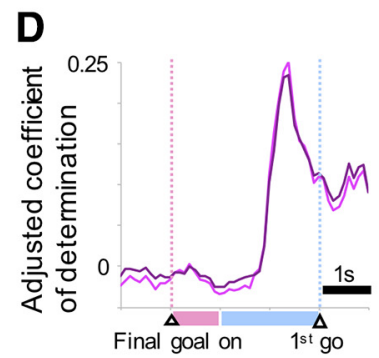

- Immediate-goal + Sequence-pattern

- Cursor-movement-sequence

Figure 8. An example of the axis-tuned immediate-goal neurons showing firing modulation by not only the immediate goals but also the sequence pattern. This neuron is the same as the pyramidal neuron shown in Figure 5. $\boldsymbol{A}$, Raster plots and spike density histograms of neuronal activity for each cursor-movement sequence. $\boldsymbol{B}-\boldsymbol{D}$, Plotted ACDs. $\boldsymbol{B}$, Immediate goals (light blue lines) versus cursor-movement sequences (magenta lines). $\boldsymbol{C}$, Sequence patterns (purple lines) versus cursor-movement sequences (magenta lines). $\boldsymbol{D}$, Immediate goals + cursor-movement sequences (dark magenta lines) versus cursor-movement sequences (magenta lines).

though many neurons maintained vector tuning in both the Final goal display period and the Delay 2 period, the plot also indicates that a considerable number of neurons exhibited changes in tuning shape from the final-goal vector tuning to immediate-goal axis tuning. Table 4 demonstrates the numbers of neurons that exhibited vector and axis tuning for the final goals in the Final goal display period. Comparing Table 4 with the first line of Table 3 revealed that axis-tuned cells were prominent in the immediate-goal coding in the Delay 2 period. The distribution of this ratio for immediate goals was significantly hider than that for final goals $\left(t_{(79)}=2.8, p=0.0073\right.$, Cohen's $d=0.38, r=0.21$, paired $t$ test), indicating that the axis components were increased in the immediate-goal directional tuning as a whole.

\section{Relevance to cursor-movement sequences}

The path-planning task requires a stepwise cursor-movement sequence to reach a final goal. In this section, the relevance of the immediate-goal neurons to cursor-movement sequences was examined. Figure $8 \mathrm{~A}$ displays the raster and histograms of the pyramidal neuron shown in Figure 5 replotted for the cursormovement sequences. As shown in Figure 5, this neuron did not fire well for the cases in which the immediate goal was left or right. However, in cases of up or down immediate goals, the firing rates seemed modulated. This neuron apparently exhibited higher activities for trials of up $\rightarrow$ up $\rightarrow$ left, up $\rightarrow$ up $\rightarrow$ right, down $\rightarrow$ down $\rightarrow$ left, and down $\rightarrow$ down $\rightarrow$ right than trials of up $\rightarrow$ left $\rightarrow$ up, up $\rightarrow$ right $\rightarrow$ up, down $\rightarrow$ left $\rightarrow$ down, and down $\rightarrow$ right $\rightarrow$ down. That is, the neuronal activities were seemingly modulated by the abstract sequence patterns, such as XXY, XYX, and YXX (Fig. 2).

To quantify these tendencies, a regression analysis was performed for the cursor-movement sequences by using regressors composed of three types of subfactors: the immediate goals, sequence patterns, and residual factors necessary to reach the final goal (see Materials and Methods; Fig. 2). As observed in Figure $8 B$, although the ACD for the immediate goals (blue line) exhibited high values in the Delay 2 period alone, it was inferior to the ACD for cursor-movement sequences (Fig. 8B, C,D, magenta lines). When analyzed using only the regressors of the sequence patterns (Fig. $8 C$, purple line), even though the ACD was also elevated during the Delay 2 period, it was lower than the ACDs not only for the cursor-movement sequences but also for immediate goals. In contrast to Figure $8 B$, $C$, Figure $8 D$ demonstrates that the ACD line obtained using the regressors for the immediate goals and sequence patterns (dark magenta line; i.e., without the residual factor) almost overlapped with that for cursormovement sequences; this indicates that most of the pyramidal 


\section{Axis-tuned immediate-goal neurons}
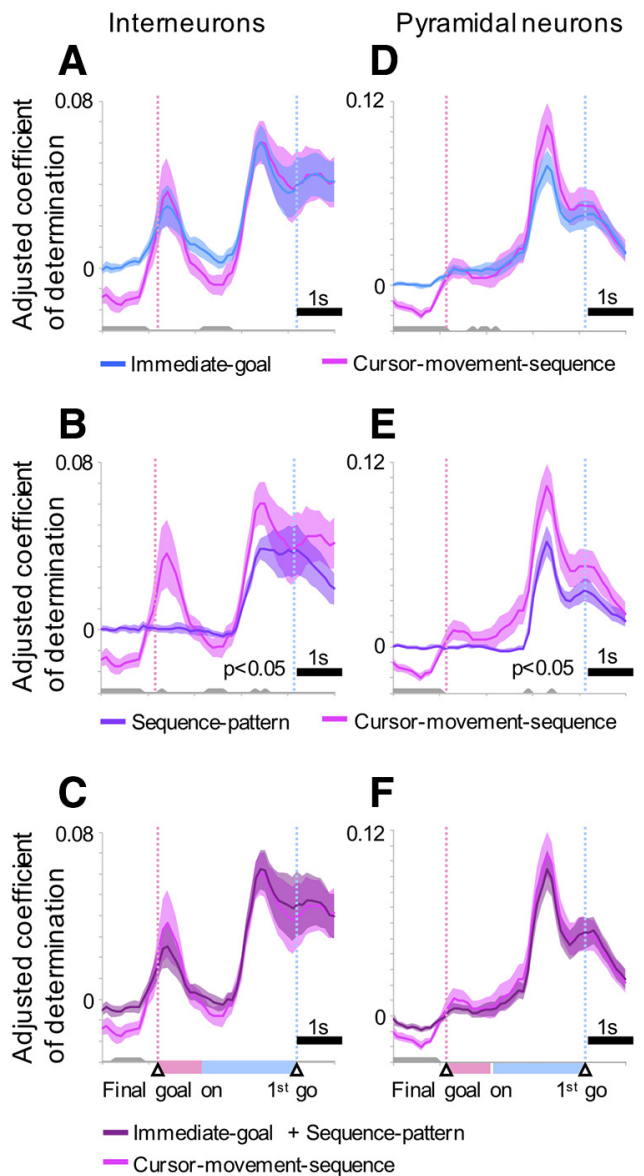

Figure 9. Comparison of the firing modulation between the cursor-movement sequences and related parameters in the axis-tuned immediate-goal neurons. ACDs are shown. $\boldsymbol{A}-\boldsymbol{C}$, Interneurons $(n=20)$. $\boldsymbol{D}-\boldsymbol{F}$, Pyramidal neurons $(n=44)$. $\boldsymbol{A}, \boldsymbol{D}$, Immediate goals (light blue lines) versus cursor-movement sequences (magenta lines). $\boldsymbol{B}, \boldsymbol{E}$, Sequence patterns (purple lines) versus cursor-movement sequences (magenta lines). $\boldsymbol{C}, \boldsymbol{F}$, Immediate goals + cursormovement sequences (dark magenta lines) versus cursor-movement sequences (magenta lines). Gray bars below the graphs represent periods showing significant differences $(p<0.05$, Wilcoxon rank-sum test). Shaded areas represent SEM.

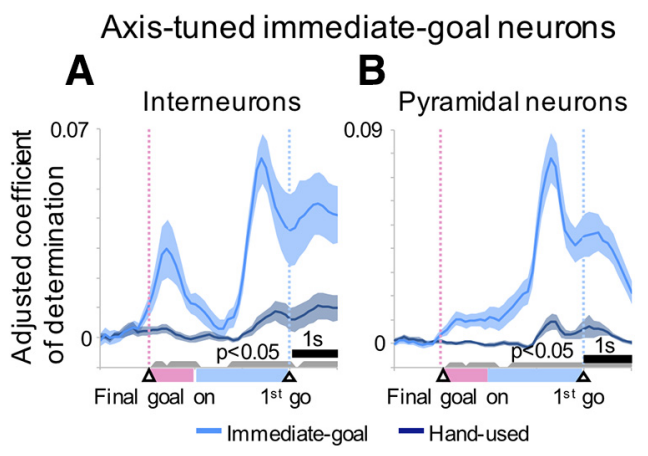

Figure 10. Comparison of the firing modulation between immediate goals and the hand used by the monkey to move the cursor in the axis-tuned immediate-goal neurons. ACDs for the immediate goals (light blue line) and the hand used (dark blue line) are shown. $\boldsymbol{A}$, Interneurons $(n=20)$. $\boldsymbol{B}$, Pyramidal neurons $(n=44)$. Periods showing significant differences are designated by gray bars below the graphs ( $p<0.05$, Wilcoxon rank-sum test). Shaded areas represent SEM.

\section{Reaction-time of the first movement}

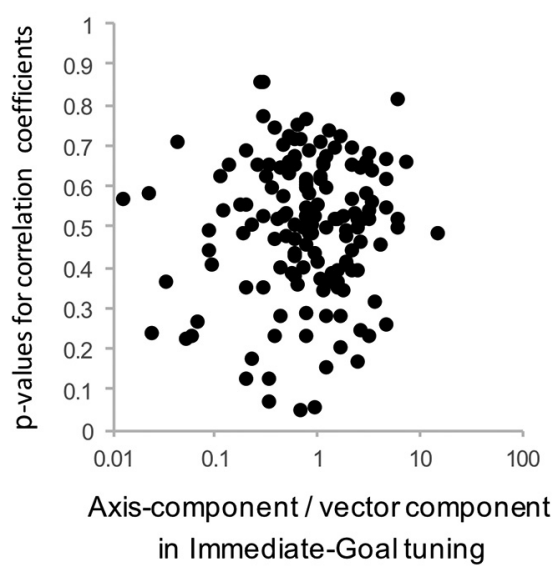

Figure 11. The $p$ values of correlation coefficients between reaction times for the first movement and spike counts at the peak of immediate-goal selectivity plotted against the ratio of the axis component to the vector component of immediate-goal tuning at the same peak. Each dot represents the $p$ value for an immediate-goal neuron $(n=148)$.

neuronal firing modulation by the cursor-movement sequences can be explained by the immediate goals and sequence patterns.

The properties of the pyramidal neuron shown in Figure 8 were recognized at the population level (Fig. 9). For interneurons with an axis tuning of immediate goals $(n=20)$, the ACD for the immediate goals (Fig. 9A, blue line) overlapped with that for the cursor-movement sequences (Fig. $9 A-C$, magenta lines) and did not show any significant differences during the Delay 2 period ( $p=0.70$ at minimum, Wilcoxon rank-sum test). In addition, the ACD for the sequence patterns (Fig. $9 B$, purple line) was significantly below that for the cursor-movement sequences ( $p=$ 0.036 at minimum, Wilcoxon rank-sum test). Regarding the pyramidal neurons with the axis tuning $(n=44)$, the ACD for the immediate goals was below that for the cursor-movement sequences, but not significantly (Fig. $9 D ; p=0.11$ at minimum, Wilcoxon rank-sum test). Neither the ACD for the sequence patterns (Fig. 9E, purple line) reached the ACD for the cursormovement sequences, but unlike the case of the immediate goals, this difference was significant ( $p=0.042$ at minimum, Wilcoxon rank-sum test). When analyzed using the regressors for the immediate goals and pattern sequences (Fig. 9F), the ACD (dark magenta line) overlapped well with that for the cursormovement sequence, not only during the Delay 2 period ( $p=$ 0.60 at minimum, Wilcoxon rank-sum test) but also before and after it. This result demonstrated that, in these pyramidal neurons, the firing modulations in different cursor-movement sequences could be well explained by the modulations caused by changes in immediate goals and sequence patterns.

\section{Relation to other behavioral parameters}

We considered that the immediate-goal axial tuning seen in the Delay 2 period described above may merely reflect other behavioral parameters. To test this, we performed regression and correlation analyses on several parameters, as follows.

In our task paradigm, up-down and left-right cursor movements were executed by the left and right hand in Assignments 1 and 2, respectively; this might have appeared as axis tuning. However, the mean hand-used selectivities of both the interneurons $(n=20)$ and pyramidal neurons $(n=44)$ were not significant $(p=0.05)$ throughout the preparatory period. Figure $10 A, B$ also indicated that the ACDs for hand used (dark blue) were signifi- 


\section{Vertical eye-position}
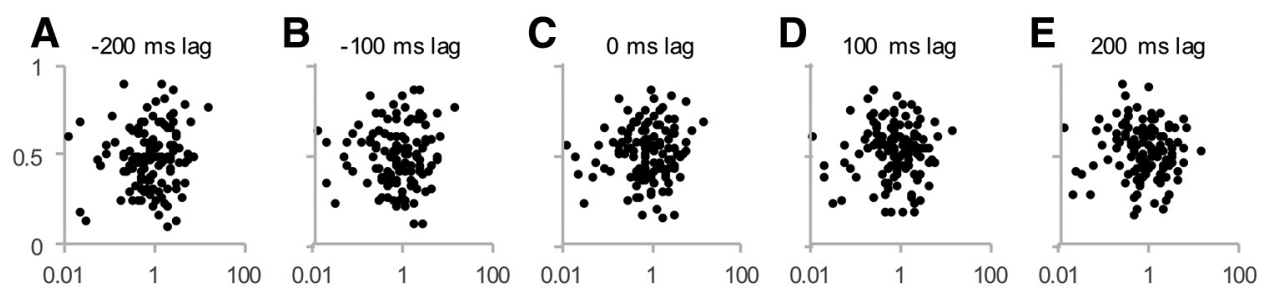

Horizontal eye-position
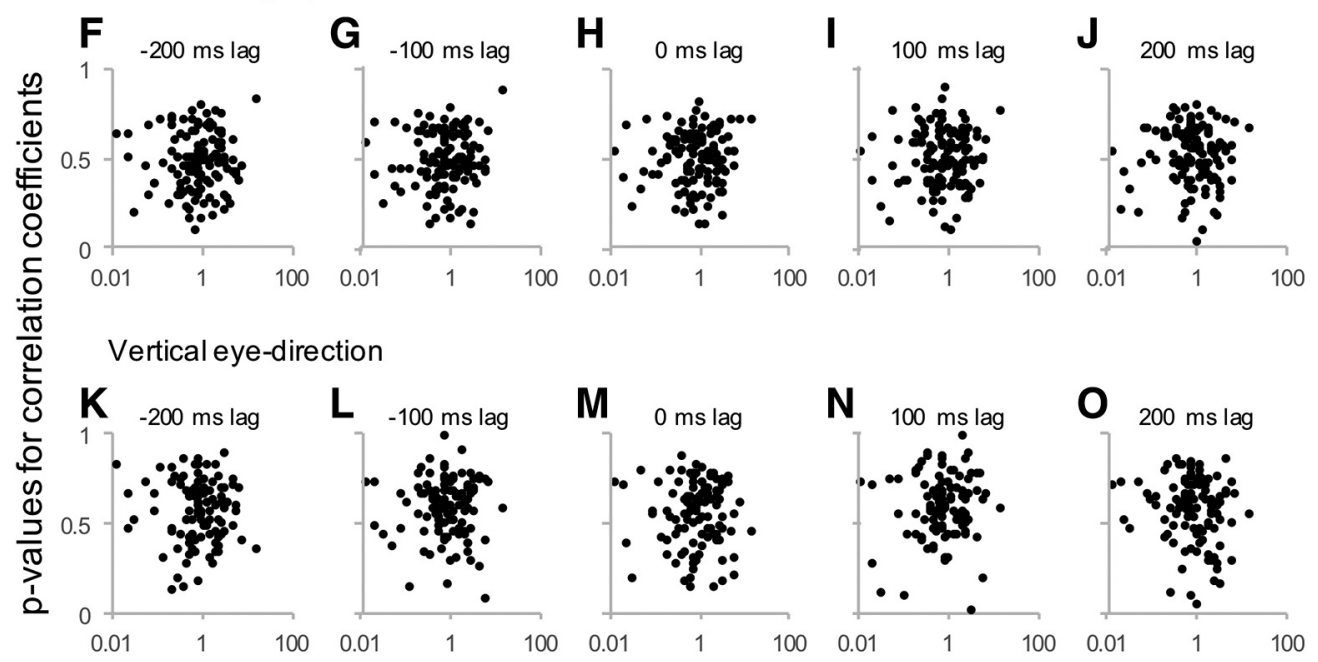

Horizontal eye-direction
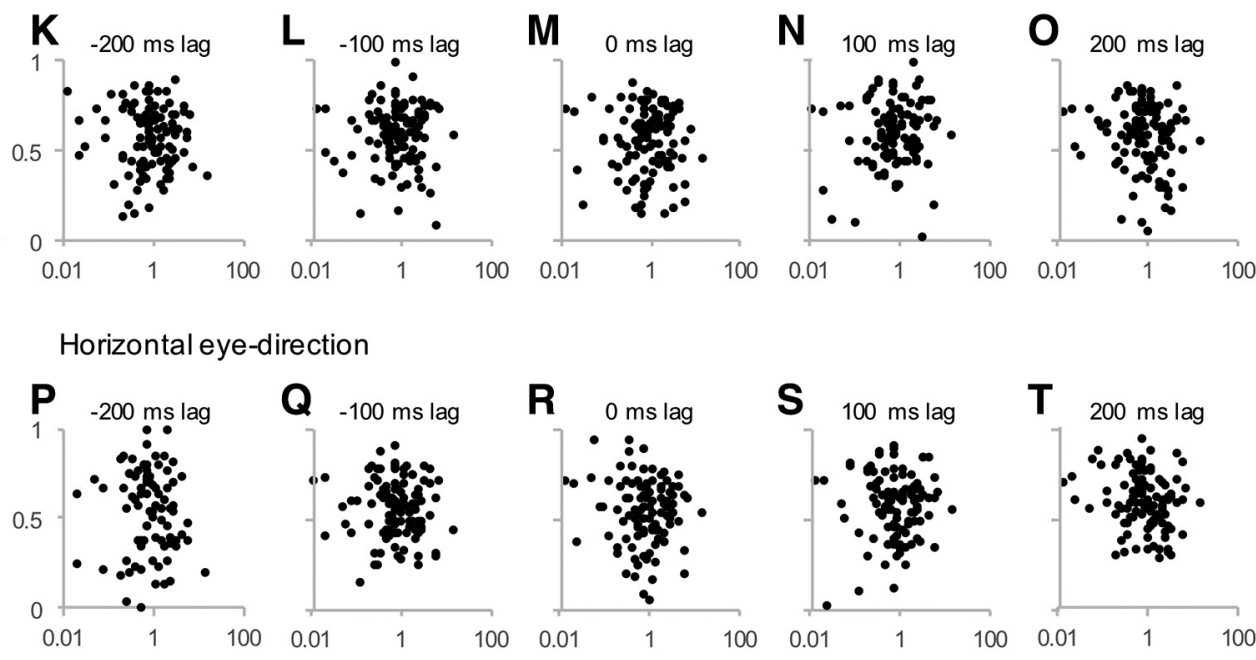

\section{Axis-component/ vector component in Immediate-Goal tuning}

Figure 12. The $p$ values of correlation coefficients between eye movements and spike counts at the peak of immediate-goal selectivity plotted against the ratio of the axis component to the vector component of the immediate-goal tuning. $\boldsymbol{A}-\boldsymbol{E}$, Vertical eye position. $\boldsymbol{F}-\boldsymbol{J}$, Horizontal eye position. $\boldsymbol{K}-\mathbf{O}$, Vertical eye-direction. $\boldsymbol{P}-\boldsymbol{T}$, Horizontal eye-direction. From left to right, Correlations for $-200,-100,0,100$, and $200 \mathrm{~ms}$ time lags between eye movements and the peak immediate-goal selectivity. Each dot represents the $p$ value of an immediate-goal neuron ( $n=148)$.

cantly smaller than those for the immediate goal (light blue) $(p<$ 0.05 , Wilcoxon rank-sum test) during most of the preparatory period in both interneurons and pyramidal neurons. These results indicated that the neuronal activities of the immediate-goal neurons reflected the "cognitive" cursor movements not hand "muscular" movements.

Next, we calculated the correlation between reaction times and spike counts at the peak of the immediate-goal selectivity for each immediate-goal neuron. Figure 11 illustrates the relationships between the $p$ values for correlation coefficients and ratios of axis components to vector components of immediate-goal tuning, at the peak of the immediate-goal selectivity for each neuron. Importantly, almost all of the correlations were nonsignificant, indicating that immediate-goal selectivity is not related to reaction time.

We also considered that vector or axis tuning of the immediate-goal neurons may merely reflect eye movement. To test this, we examined correlations of eye movements and spike counts at the peak of the immediate-goal selectivity. Correlation coefficients were obtained for four eye movement parameters: horizontal and vertical positions and directions. These were calculated for five time lags $(-200,-100,0,100$, and $200 \mathrm{~ms})$ between the peak of the immediate-goal selectivity and eye movements, which covered the period in which changes in visual information due to eye movements affected neuronal activities, and the period in which neuronal activities elicited eye movement; however, our dataset did not include data on neurons from the frontal eye field (Saito et al., 2005). In Figure 12, $p$ values for these correlations are plotted against the ratio of the axis component to vector component, for the immediate-goal tuning at the peak immediate-goal selectivity for each neuron, in the same manner as in Figure 11. As demonstrated by these 20 plots, few correlations were statistically significant. Thus, we concluded that the immediate-goal selectivity of these neurons did not merely reflect the influence of eye movements on their firing activities. 


\section{Discussion}

The present study examined the tuning shapes of IPFC neurons that were associated with the four directions of each of the final goals and immediate goals while monkeys performed a path-planning task. Many neurons exhibited axis tuning for immediate goals before the execution of a cursor movement. Moreover, axis tuning was dynamically transformed from vector tuning for final goals in many of those neurons. The activities of these neurons, especially pyramidal neurons, were also modulated by the abstract sequence patterns. Such a dynamic coding strategy of the IPFC might contribute to the flexible adaption of behavior in changeable environments with limited neuronal resources.

The existence of axis tuning for direction in monkey IPFC neurons

In this study, a large number of immediate-goal neurons showed axis tuning for immediate-goal directions: the neurons fired well in response to both up and down or both left and right immediate goals in the Delay 2 period (Figs. $3 C, 4 C$, $5 C)$. The time development of the axis component of firing rate (Figs. $3 G, 4 G$, $5 G, 6 G, I)$ and the scatter plot of the ratio of the axis component to the vector component (Fig. 7) also verified the relative increase of axis tuning over vector tuning in the immediate-goal selectivity. Comparison of time developments between immediate-goal selectivity (Figs. $3 B, 4 B$, $5 B, 6 A, B$, blue lines) and the axis component of the firing rate for immediate goals (Figs. 3G, 4G, 5G, 6G,I, blue lines) demonstrated the strong relationship between immediate-goal selectivity and axis tuning of the immediate goals.

This axis tuning cannot be regarded as a mere reflection of the behavioral parameters described in the following. Up-down and left-right cursor movements were executed by the left and right hands in Assignments 1 and 2, respectively. This suggests that the axis tuning of immediategoal neurons was dependent on the hand used. However, regression analysis demonstrated that the hands used did not significantly influence the observed neuronal activities (Fig. 10A,B). We also confirmed that the immediate-goal selectivity was not merely a reflection of reaction time (Fig. 11), nor was it a reflection of the effects of eye movements on neuronal firing activities (Fig. 12). We did not examine neural activities in anticipation of the Go signal. The Go signal was delivered in every trial; thus, neuronal activities corre-
A

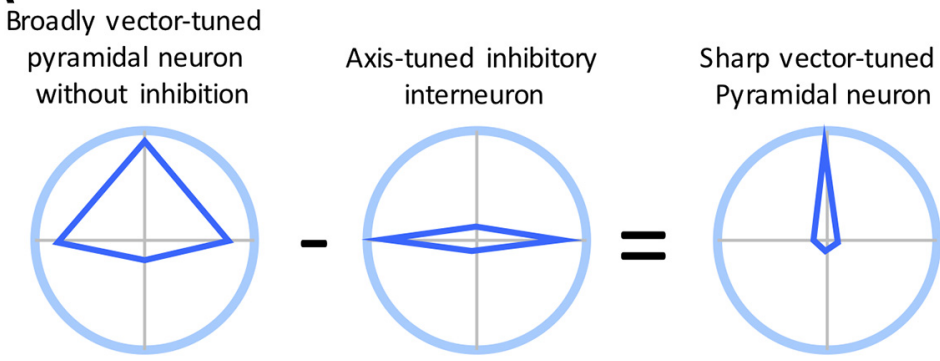

B

Non selective pyramidal neuron without inhibition

Axis-tuned inhibitory interneuron
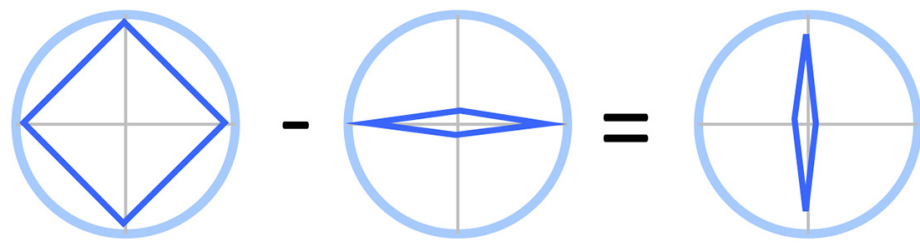

Figure 13. One possible mechanism of the case in which axis-tuned cells are observed mainly in interneurons. $A$, In the example shown in the left figure, the pyramidal neuron has a preferred direction (in this example, "up"); however, it also fires well to the both sides of the preferred direction (in this case, "left" and "right") without inhibition. If an axis-tuned interneuron that exhibits strong activities for "left" and "right" directions (middle) exists adjacent to the pyramidal cell and inhibits it, the tuning of the pyramidal neuron is shaped as demonstrated at right. $\boldsymbol{B}$, As a byproduct, axis-tuned pyramidal neurons can emerge. That is, if a pyramidal neuron with no directional tuning (left) is inhibited by an axis-tuned inhibitory interneuron (middle), the pyramidal neuron can exhibit axis tuning (right).

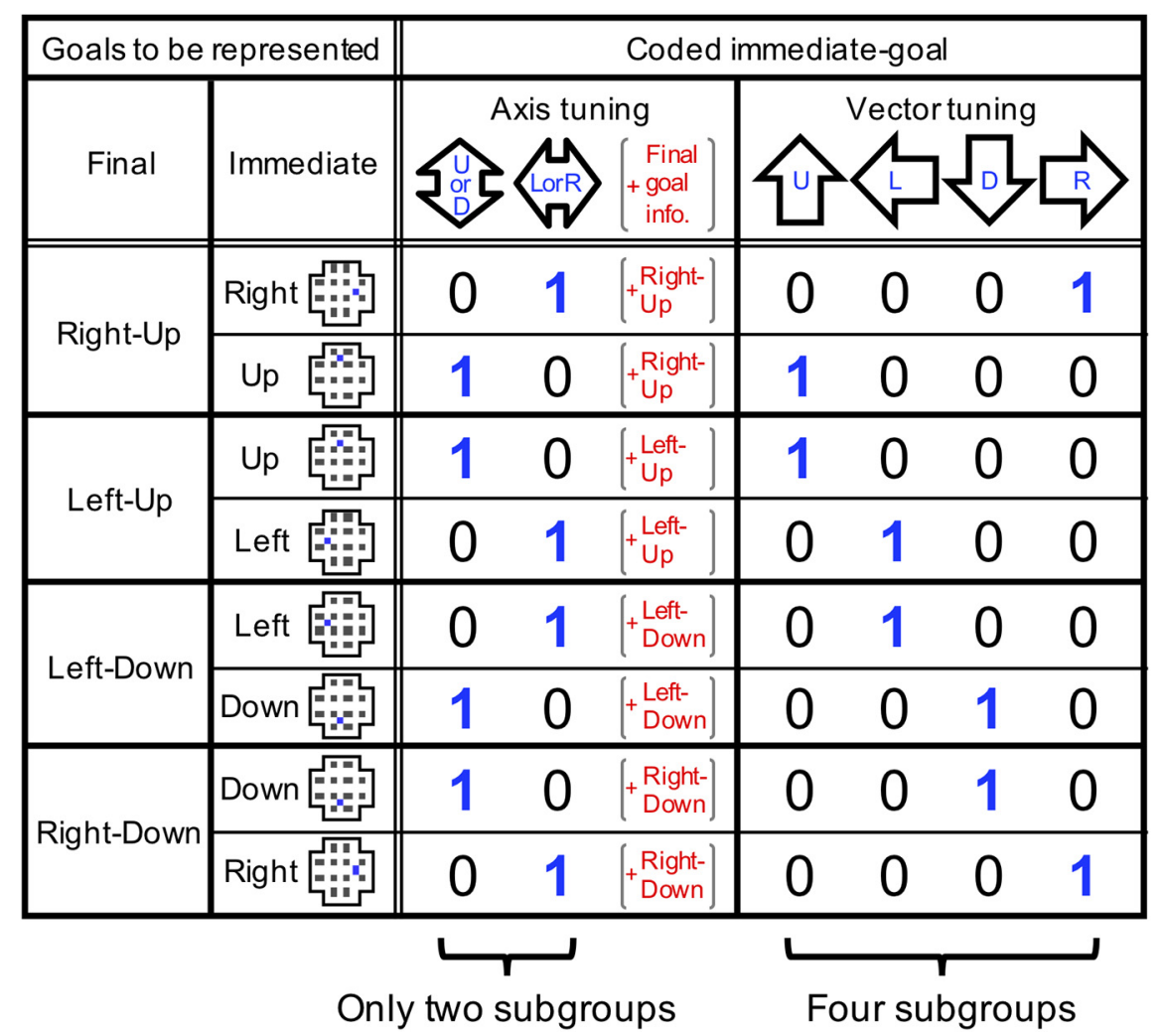

Figure 14. A possible case in which a significant number of pyramidal neurons sends the information of immediate goals to remote neurons with axis tuning. The table exhibits possible schemes for coding immediate goals. Each single-headed or doubleheaded arrow indicates the neural group that encodes the immediate goal written within the arrow. " 0 " and " 1 " represent "on" and "off" for each the neuronal group, respectively. The middle column demonstrates that a particular immediate-goal direction can be decoded from only two populations of axis-tuned cells coding immediate goals, with the aid of the final-goal neurons coding the direction of the final goal. 


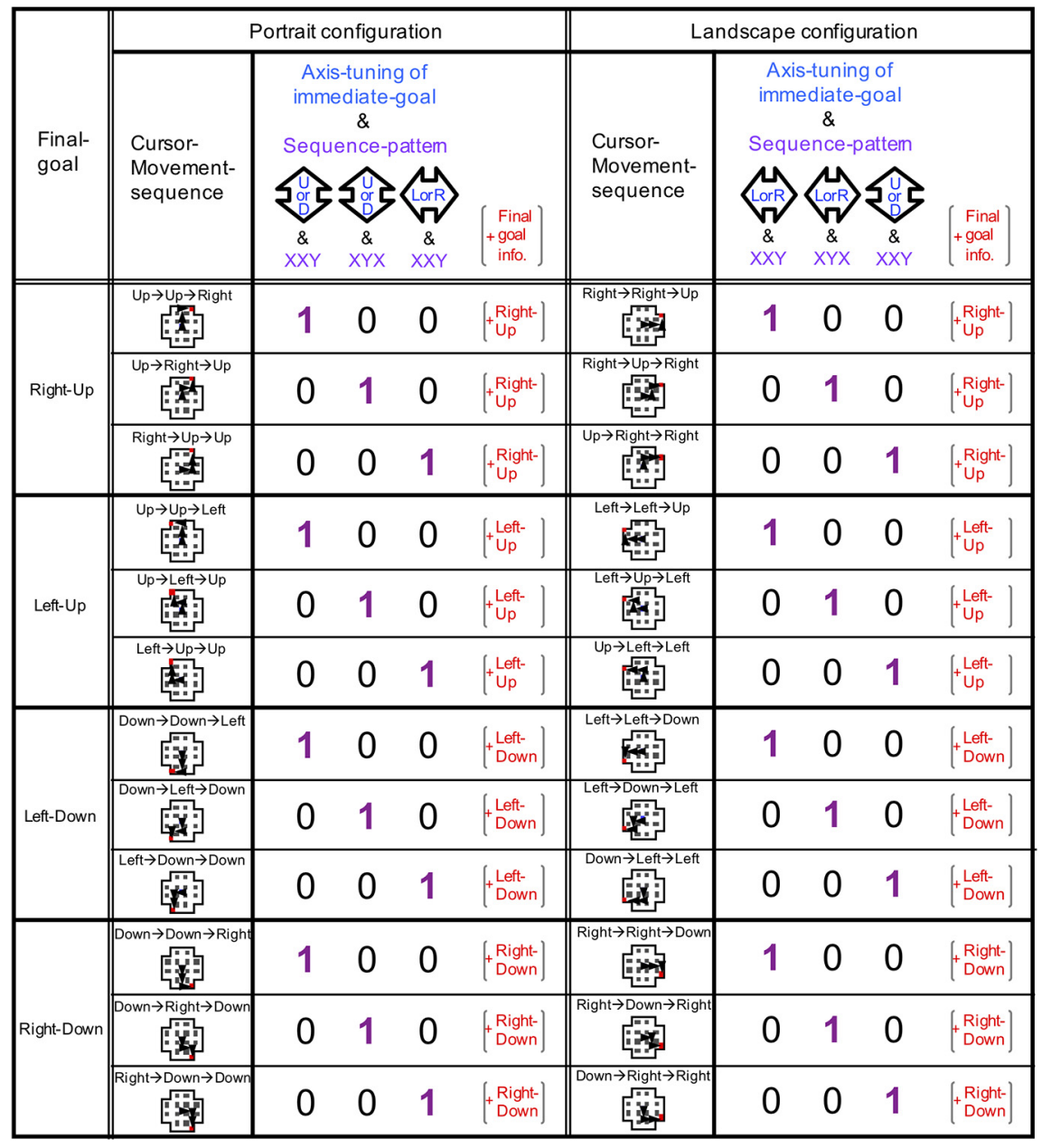

Figure 15. One possible scheme for representation of the cursor-movement sequence by neuronal populations coding immediate goals with axis tuning and sequence patterns. The figure formats follow those in Figure 14. ity of pyramidal neurons (Fig. $6 B$, red line) during the Delay 1 period suggests that the pyramidal neurons retain finalgoal information, such as that available for remote neurons, whereas the reduction in final-goal selectivity in interneurons (Fig. $6 A$, red line) reflects the involvement in internal processes within local circuits.

\section{Significance of axis tuning in path-planning tasks}

What is the functional meaning of axis tuning of immediate goals? If axis cells were observed mainly in interneurons, axis cells would be interpreted as reflecting an intermediate state of the immediategoal-encoding process. For example, it seems possible that sharp vector tuning of pyramidal cells is formed by suppressing their activities via axis-tuned interneurons (Fig. 13A). Furthermore, as an intermediate byproduct of the process, some pyramidal neurons may exhibit axis tuning. That is, pyramidal cells without directional tuning in the absence of suppression can indicate axis tuning by being suppressed by inhibitory interneurons with axis tuning (Fig. 13B).

The observation of axis tuning in a substantial number of pyramidal neurons that can project to remote areas raises the question of how a remote neuron can decode the information of immediate goal coded by the activities of axis-tuned neurons. Vector tuning, in which each immediate goal is represented by the activities of four distinct neuronal groups coding up, sponding to Go-signal anticipation would not be modulated by goal direction. The current study focused on neurons that exhibited some significant directional components, such that neurons showing only a nondirectional elevation in firing rate before the Go signal were excluded.

Recent studies identified axis-tuned or bilateral directional cells in the subiculum and retrosplenial cortex of rats (Jacob et al., 2017; Olson et al., 2017). These brain areas are components of the hippocampal navigation system and have strong anatomical interactions with the IPFC in nonhuman primates (GoldmanRakic et al., 1984; Morris et al., 1999). Thus, although the existence of axis-tuned cells in the monkey IPFC might have been predictable, the present study was the first to report it.

\section{Distinction of putative interneurons and pyramidal neurons}

Distinguishing between interneurons and pyramidal neurons provides good insight into the processing occurring within the area where neural activity is recorded. In this study, interneurons and pyramidal neurons were carefully distinguished based on waveform, and the waveform widths and firing rates were consistent with those reported in previous IPFC studies (Wilson et al., 1994; Rao et al., 1999; Constantinidis and Goldman-Rakic, 2002; Diester and Nieder, 2008; Hussar and Pasternak, 2009, 2012; Johnston et al., 2009; Qi et al., 2011). Interneurons form synapses with adjacent neurons only, whereas pyramidal neurons project to distant neurons as well. Thus, the sustained final-goal selectivdown, left, and right, appears natural in the sense that each neuronal group solely drives downstream neurons without requiring specific mechanisms for reading out the immediate-goal information. In contrast, there are only two subtypes of immediategoal neurons with axis tuning: one prefers up and down immediate goals and the other left and right immediate goals. The activity of each subtype does not, by itself, distinguish between the two immediate goals. However, as depicted in Figure 14, by considering the final-goal neuronal groups that represent final goals, it is possible to identify a single immediate goal.

IPFC neuronal activities reflect the behavioral sequences to be executed (e.g., Averbeck et al., 2006; Averbeck and Lee, 2007). Consistent with this, the activities of the pyramidal cells among the axis-tuned immediate-goal neurons were largely modulated by the cursor-movement sequences, as demonstrated in Figures 8 and 9. The existence of the axis tuning of the immediate goals was also confirmed repeatedly in this study. This raises the question of how we can understand these seemingly unrelated facts in the IPFC. Another finding of this study that most of the firing modulation by the cursor-movement sequences was explained by the combination of the immediate goals and abstract sequence patterns may be key (Figs. $8 D, 9 F$ ). The abstract representation of movement sequence by the IPFC neurons was originally reported by Shima et al. (2007). Figure 15 provides a possible explanation for the coexistence of axis tuning and sequence patterns within IPFC neurons. Specifically, many possible cursor-movement se- 
quences in the path-planning task (12 each for the portrait and the landscape configurations) can be coded by a small number of the neuronal populations (three each for the two configurations), each of which represents the combination of the axis-tuned immediate-goals and the abstract sequence pattern, with the aid of the final-goal neurons representing the direction of the final goals.

These ideas regarding efficient usage of neuronal resources will require further discussion. However, axis-tuned cells were also reported in the rodent subiculum (Olson et al., 2017) and retrosplenial cortex (Jacob et al., 2017). Although discussions of the rat subiculum and retrosplenial cortex and monkey IPFC should not be intermingled, axis-tuned cells in these brain areas may be involved in the abstract and efficient representation of movement sequences.

\section{Dynamic usage of neuronal resources in the IPFC to adapt to an ever-changing environment}

The present study demonstrated that a group of neurons in the IPFC exhibited axis tuning in response to immediate goals, and that axis tuning for immediate goals was dynamically transformed from vector tuning for final goals along the time course of the preparatory period of the task. These results suggest that IPFC neurons change not only what to code but also how to code dynamically during behavioral planning. This flexibility appears plausible given the nature of executive functions, which are "the high-level cognitive processes that facilitate new ways of behaving, and optimize one's approach to unfamiliar circumstances" (Gilbert and Burgess, 2008). A wide variety of activities, from sustained activities involved in working memory (Funahashi et al., 1989; Miller et al., 1996; Romo et al., 1999) to activities related to planned action sequences (Barone and Joseph, 1989; Averbeck et al., 2002, 2006; Mushiake et al., 2006; Averbeck and Lee, 2007; Sigala et al., 2008), may be a reflection of the flexibility of the IPFC. Such flexibility is considered to emerge within the IPFC itself. Sakamoto et al. (2013) provided unique evidence for increased firing variability, as a "critical fluctuation" (Schöner and Kelso, 1988; Chen et al., 2012; Scheffer et al., 2009), before transformation of the goal representation, suggesting that dynamic coding of the IPFC is not caused by altered input, but by state transition of the network itself. To adapt to complex, unpredictable, and ever-changing environments, the IPFC neurons may autonomously switch the contents they code as well as the way they code. Such dynamic usage of neuronal resources in the IPFC must reflect the critical nature of executive functions.

\section{References}

Averbeck BB, Lee D (2007) Prefrontal neural correlates of memory for sequences. J Neurosci 27:2204-2211.

Averbeck BB, Chafee MV, Crowe DA, Georgopoulos AP (2002) Parallel processing of serial movements in prefrontal cortex. Proc Natl Acad Sci U S A 99:13172-13177.

Averbeck BB, Sohn JW, Lee D (2006) Activity in prefrontal cortex during dynamic selection of action sequences. Nat Neurosci 9:276-282.

Barone P, Joseph JP (1989) Prefrontal cortex and spatial sequencing in macaque monkey. Exp Brain Res 78:447-464.

Chen L, Liu R, Liu ZP, Li M, Aihara K (2012) Detecting early-warning signals for sudden deterioration of complex diseases by dynamical network biomarkers. Sci Rep 2:342.

Constantinidis C, Goldman-Rakic PS (2002) Correlated discharges among putative pyramidal neurons and interneurons in the primate prefrontal cortex. J Neurophysiol 88:3487-3497.

Diester I, Nieder A (2008) Complementary contributions of prefrontal neuron classes in abstract numerical categorization. J Neurosci 28: $7737-7747$.
Draper NR, Smith H (1998) Applied regression analysis. New York: Wiley.

Duncan J (2001) An adaptive coding model of neural function in prefrontal cortex. Nat Rev Neurosci 2:820-829.

Freedman DJ, Riesenhuber M, Poggio T, Miller EK (2001) Categorical representation of visual stimuli in the primate prefrontal cortex. Science 291:312-316

Funahashi S, Bruce CJ, Goldman-Rakic PS (1989) Mnemonic coding of visual space in the monkey's dorsolateral prefrontal cortex. J Neurophysiol 61:331-349.

Genovesio A, Brasted PJ, Wise SP (2006) Representation of future and previous spatial goals by separate neural populations in prefrontal cortex. J Neurosci 26:7305-7316.

Genovesio A, Tsujimoto S, Wise SP (2012) Encoding goals but not abstract magnitude in the primate prefrontal cortex Neuron 74:656-662.

Gilbert SJ, Burgess PW (2008) Executive function. Curr Biol 18: R110-R114.

Goldman-Rakic PS, Selemon LD, Schwartz ML (1984) Dual pathways connecting the dorsolateral prefrontal cortex with the hippocampal formation and parahippocampal cortex in the rhesus monkey. Neuroscience 12:719-743.

Hussar CR, Pasternak T (2009) Flexibility of sensory representations in prefrontal cortex depends on cell type. Neuron 64:730-743.

Hussar CR, Pasternak T (2012) Memory-guided sensory comparisons in the prefrontal cortex: contribution of putative pyramidal cells and interneurons. J Neurosci 32:2747-2761.

Jacob PY, Casali G, Spieser L, Page H, Overington D, Jeffery K (2017) An independent, landmark-dominated head-direction signal in dysgranular retrosplenial cortex. Nat Neurosci 20:173-175.

Johnston K, DeSouza JF, Everling S (2009) Monkey prefrontal cortical pyramidal and putative interneurons exhibit differential patterns of activity between prosaccade and antisaccade tasks. J Neurosci 29:5516-5524.

Katori Y, Sakamoto K, Saito N, Tanji J, Mushiake H, Aihara K (2011) Representational switching by dynamical reorganization of attractor structure in a network model of the prefrontal cortex. PLoS Comput Biol 7:e1002266.

Machens CK, Romo R, Brody CD (2005) Flexible control of mutual inhibition: a neural model of two-interval discrimination. Science 307:11211124

Mante V, Sussillo D, Shenoy KV, Newsome WT (2013) Context-dependent computation by recurrent dynamics in prefrontal cortex. Nature 503:78 84.

Markram H, Toledo-Rodriguez M, Wang Y, Gupta A, Silberberg G, Wu C (2004) Interneurons of the neocortical inhibitory system. Nat Rev Neurosci 5:793-807.

Miller EK, Erickson CA, Desimone R (1996) Neural mechanisms of visual working memory in prefrontal cortex of the macaque. J Neurosci 16: $5154-5167$.

Morris R, Pandya DN, Petrides M (1999) Fiber system linking the meddorsolateral frontal cortex with the retrosplenial/presubicular region in the rhesus monkey. J Comp Neurol 407:183-192.

Murray JD, Bernacchia A, Roy NA, Constantinidis C, Romo R, Wang XJ (2017) Stable population coding for working memory coexists with heterogeneous neural dynamics in prefrontal cortex. Proc Natl Acad Sci U S A 114:394-399.

Mushiake H, Saito N, Sakamoto K, Sato Y, Tanji J (2001) Visually based path planning by Japanese monkeys. Brain Res Cogn Brain Res 11:165169.

Mushiake H, Saito N, Sakamoto K, Itoyama Y, Tanji J (2006) Activity in the lateral prefrontal cortex reflects multiple steps of future events in action plans. Neuron 50:631-641.

Olson JM, Tongprasearth K, Nitz DA (2017) Subiculum neurons map the current axis of travel. Nat Neurosci 20:170-172.

Passingham RE, Wise SP (2012) The neurobiology of the prefrontal cortex: anatomy, evolution, and the origin of insight. Oxford: Oxford UP.

Qi XL, Meyer T, Stanford TR, Constantinidis C (2011) Changes in prefrontal neuronal activity after learning to perform a spatial working memory task. Cereb Cortex 21:2722-2732.

Rao SG, Williams GV, Goldman-Rakic PS (1999) Isodirectional tuning of adjacent interneurons and pyramidal cells during working memory: evidence for microcolumnar organization in PFC. J Neurophysiol 81: 1903-1916.

Rigotti M, Barak O, Warden MR, Wang XJ, Daw ND, Miller EK, Fusi S 
(2013) The importance of mixed selectivity in complex cognitive tasks. Nature 497:585-590.

Romo R, Brody CD, Hernández A, Lemus L (1999) Neuronal correlates of parametric working memory in the prefrontal cortex. Nature 399:470-473.

Saito N, Mushiake H, Sakamoto K, Itoyama Y, Tanji J (2005) Representation of immediate and final behavioral goals in the monkey prefrontal cortex during an instructed delay period. Cereb Cortex 15:1535-1546.

Sakamoto K, Mushiake H, Saito N, Aihara K, Yano M, Tanji J (2008) Discharge synchrony during the transition of behavioral goal representations encoded by discharge rates of prefrontal neurons. Cereb Cortex 18: 2036-2045.

Sakamoto K, Katori Y, Saito N, Yoshida S, Aihara K, Mushiake H (2013) Increased firing irregularity as an emergent property of neural-state transition in monkey prefrontal cortex. PLoS One 8:e80906.

Scheffer M, Bascompte J, Brock WA, Brovkin V, Carpenter SR, Dakos V, Held H, van Nes EH, Rietkerk M, Sugihara G (2009) Early-warning signals for critical transitions. Nature 461:53-59.

Schöner G, Kelso JA (1988) Dynamic pattern generation in behavioral and neural systems. Science 239:1513-1520.
Shima K, Isoda M, Mushiake H, Tanji J (2007) Categorization of behavioural sequences in the prefrontal cortex. Nature 445:315-318.

Sigala N, Kusunoki M, Nimmo-Smith I, Gaffan D, Duncan J (2008) Hierarchical coding for sequential task events in the monkey prefrontal cortex. Proc Natl Acad Sci U S A 105:11969-11974.

Tanji J, Hoshi E (2008) Role of the lateral prefrontal cortex in executive behavioral control. Physiol Rev 88:37-57.

Wallis JD, Anderson KC, Miller EK (2001) Single neurons in prefrontal cortex encode abstract rules. Nature 411:953-956.

Wilson FA, O'Scalaidhe SP, Goldman-Rakic PS (1994) Functional synergism between putative gamma-aminobutyrate-containing neurons and pyramidal neurons in prefrontal cortex. Proc Natl Acad Sci U S A 91:4009-4013.

Wörgötter F, Eysel UT (1987) Quantitative determination of orientational and directional components in the response of visual cortical cells to moving stimuli. Biol Cybern 57:349-355.

Wörgötter F, Eysel UT (1991) Axial responses in visual cortical cells: spatiotemporal mechanisms quantified by Fourier components of cortical tuning curves. Exp Brain Res 83:656-664. 